DCPT- $05 / 05$

hep-th/0502195

\title{
Black hole thermodynamics
}

\author{
Simon F. Ross* \\ Centre for Particle Theory, Department of Mathematical Sciences \\ University of Durham, South Road, Durham DH1 3LE, U.K.
}

\begin{abstract}
These notes introduce basic aspects of black hole thermodynamics. I review the classical laws of black hole mechanics, give a brief introduction to the essential concepts of quantum field theory in curved spacetime, and derive the Unruh and Hawking effects. I conclude with a discussion of entropy from the Euclidean path integral point of view and in the context of the AdS/CFT correspondence. Originally delivered as a set of lectures at the PIMS summer school "Strings, Gravity and Cosmology" in August 2004.
\end{abstract}

*S.F.Ross@durham.ac.uk 


\section{Contents}

1 Introduction 1

2 Review of black holes 3

2.1 Horizons . . . . . . . . . . . . . . . . . . . . . 7

2.2 Near-horizon structure . . . . . . . . . . . . . . . . . . 8

3 Classical black hole thermodvnamics 9

3.1 Area theorem . . . . . . . . . . . . . . . . . . . . 9 9

3.2 Zeroth and first laws . . . . . . . . . . . . . . . . . . . 10

4 Quantum field theory in curved spacetime 14

4.1 Canonical quantisation . . . . . . . . . . . . . . . . 15

4.2 Peierls bracket . . . . . . . . . . . . . . . . . . . . . . . . . . . . . . . . . . . 17

4.3 Observables \& Hadamard states . . . . . . . . . . . . . . . . . . . . . 19

4.4 KMS condition . . . . . . . . . . . . . . . . . . . . . . . . . . . . . . 20

5 Unruh \& Hawking radiation 23

5.1 Unruh radiation . . . . . . . . . . . . . . . . . . . . . . 23

5.2 Hawking radiation . . . . . . . . . . . . . . . . . 27

$\begin{array}{lll}6 & \text { Euclidean entropy calculation } & 31\end{array}$

\section{Introduction}

The aim of these notes is to provide a brief introduction to the basic ideas of black hole thermodynamics. The principal aim is to explain the association of a temperature and entropy with stationary black holes,

$$
T=\frac{\hbar \kappa}{2 \pi}, \quad S=\frac{A}{4 G \hbar},
$$

where $\kappa$ is the black hole's surface gravity and $A$ is the area of the event horizon. This is not intended as a review article; this is a large subject, and there are aspects I am not expert enough to review. It will reflect my own perspective, filtered through the desire to present a hopefully useful pedagogical introduction. I will focus on providing an explanation of the key results in the simplest possible contexts. Two points I intend to highlight are the universality of the results - the relations (1.1) apply to any stationary black hole ${ }^{1}$ - and the central role of regularity at the event horizon in the derivation of the thermodynamic properties. I will emphasise the Euclidean point of view on black hole thermodynamics.

\footnotetext{
${ }^{1}$ This is for Einstein's general relativity. To extend it to theories with higher-curvature terms in the Lagrangian, the expression for the entropy in (1.1) is replaced by a more general integral over the event horizon, as described in section 3.2
} 
Other useful introductory reviews are 1, 2, 3, 4, 5, 6, An in-depth discussion of the derivation of Hawking radiation is given in [7, and the connection between Killing horizons and thermal properties is discussed at length in [8]. Most monographs on quantum field theory in curved spacetime include some discussion of black hole thermodynamics (see the list of references later in the introduction): a particularly comprehensive discussion is given in [9]. The recent textbook [10] also has a nice treatment of the subject. For detailed discussion of black holes and string theory, see [11, 12].

Black hole thermodynamics has continued to fascinate researchers over the 30 years since Hawking's discovery of the thermal radiation from black holes [13, 14] because it provides a real connection between gravity and quantum mechanics. The relation between geometrical properties of the event horizon and thermodynamic quantities provides a clear indication that there is a relation between properties of the spacetime geometry and some kind of quantum physics. The thermodynamic behaviour (1.1) should have a statistical interpretation in quantum gravity, and hence it provides clues to the form of the quantisation of gravity. In string theory in particular, the scaling of $S$ with the area was argued to imply that the fundamental theory cannot have local bulk degrees of freedom in the same way as an ordinary quantum field theory [15, 16, 17], and the understanding of these relations in a string theory context and providing them with a natural statistical explanation has been one of the driving forces in the revolution which has taken us beyond the formulation of string theory in terms of string scattering in a fixed background spacetime to a fully non-perturbative formulation of the theory through the AdS/CFT dualities (see for example the review [18]).

The study of black hole thermodynamics also played an important role in the development of quantum field theory in curved spacetime. This is a subject of considerable physical importance in its own right, playing an important role in, for example, the very early universe (see the lectures by Robert Brandenburger at this school, or the notes online from another set of lectures [19]). The study of such curved backgrounds has also expanded our understanding of quantum field theory in general.

These notes begin with a review of the relevant features of the classical black hole geometries, stressing the distinction between Killing and event horizons, in section 2. I describe the evidence for an analogy between black hole dynamics and thermodynamics at the classical level in section 3. Although this is the oldest part of the subject, new developments are still occurring. I give a basic introduction to quantum field theory in curved spacetime in section 4 , introducing the technology required to understand the argument for black hole thermodynamics. This is not meant as a complete introduction to this rich subject, and the interested reader would be well-advised to consult a more extensive discussion: there are several excellent texts [20, 21, 22, 9, 23, and reviews (e.g., [24, 25, 26, 27, 28]) which cover it; I particularly recommend the recent review by Jacobson [28, which provides a highly-accessible introduction to the modern viewpoint on this subject. In section [5, I will discuss the Unruh effect and Hawking radiation, showing how quantum fields on black hole backgrounds behave thermally. Finally, in section 6, the lectures finish with a more sketchy discussion of entropy from the Euclidean path-integral point of view and the understanding of 
black hole thermodynamics in AdS/CFT. Mainly because of time constraints, I have focused on equilibrium and quasi-equilibrium processes, and excluded any detailed discussion of dynamical issues such as the generalised second law.

I have attempted to make these notes reasonably self-contained and pedagogical. The choices of topics and emphasis naturally reflects my own interests; it was also influenced by the original audience. I have drawn heavily on the referenced sources in preparing these notes, and make no claim of originality for any of the content. I have attempted to give useful references to more detailed reviews and to the original literature; while it is impossible to give full references to all the people who have contributed to this subject, I welcome comments and corrections.

\section{Review of black holes}

Black hole thermodynamics involves many powerful general statements. However, to keep the discussion as concrete as possible, I will introduce the subject by focusing on the simplest possible examples. The simplest black hole solution is the Schwarzschild solution. This is a vacuum solution in general relativity in four spacetime dimensions, for which the metric is

$$
d s^{2}=-\left(1-\frac{2 M}{r}\right) d t^{2}+\left(1-\frac{2 M}{r}\right)^{-1} d r^{2}+r^{2}\left(d \theta^{2}+\sin ^{2} \theta d \phi^{2}\right) .
$$

This metric appears to have a singularity at $r=2 M$. However, this is only a coordinate singularity. We can see that the light cones in these coordinates are closing up as we approach $r=2 M$, so we can construct a better coordinate system in that region by following the causal structure: define new coordinates

$$
u, v=t \pm r_{*}=t \pm\left[r+2 M \ln \left(\frac{r}{2 M}-1\right)\right]
$$

SO

$$
\dot{u}, \dot{v}=\dot{t} \pm \frac{\dot{r}}{(1-2 M / r)} .
$$

Thus, ingoing null rays have $u=$ constant, while outgoing null rays have $v=$ constant. If we write the metric in coordinates $(u, r, \theta, \phi)$, we can extend it across $r=2 M$ along ingoing null rays. Similarly, the metric in coordinates $(v, r, \theta, \phi)$ can be extended across $r=2 M$ along outgoing null rays.

To cover the whole spacetime including the whole region behind the horizon, we need to introduce the Kruskal coordinates

$$
\begin{gathered}
u^{\prime}=e^{u / 4 M}=\left(\frac{r}{2 M}-1\right)^{1 / 2} e^{(r+t) / 4 M}, \\
v^{\prime}=-e^{-v / 4 M}=\left(\frac{r}{2 M}-1\right)^{1 / 2} e^{(r-t) / 4 M} .
\end{gathered}
$$

In terms of these coordinates,

$$
d s^{2}=-\frac{32 M^{3}}{r} e^{-r / 2 M} d u^{\prime} d v^{\prime}+r^{2} d \Omega_{2}
$$


where $r(u, v)$ is defined implicitly by (2.42.5). These coordinates are maximal-all geodesics either extend to infinite affine parameter without leaving this chart or meet the singularity at $r=0$. The singular surface at $r=2 M$ in the previous coordinates maps to $u^{\prime} v^{\prime}=0$, which is manifestly non-singular. On the other hand, $r=0$, which maps to $u^{\prime} v^{\prime}=-1$, is still singular: this is a curvature singularity. More generally, surfaces of constant $t$ are at $u^{\prime} / v^{\prime}=$ constant, while surfaces of constant $r$ are at $u^{\prime} v^{\prime}=$ constant. We will later need the form of the time-translation symmetry in these coordinates,

$$
\partial_{t}=\frac{1}{4 M}\left(u^{\prime} \partial_{u^{\prime}}-v^{\prime} \partial_{v^{\prime}}\right)
$$

These coordinates are depicted in figure 1, One of the primary advantages of this coordinate system is that the light cones lie at 45 degrees throughout the figure. This allows us to immediately see that $r=2 M$ plays a special role. Any observer who enters the region $r<2 M$ will inevitably encounter the singularity at $r=0$.

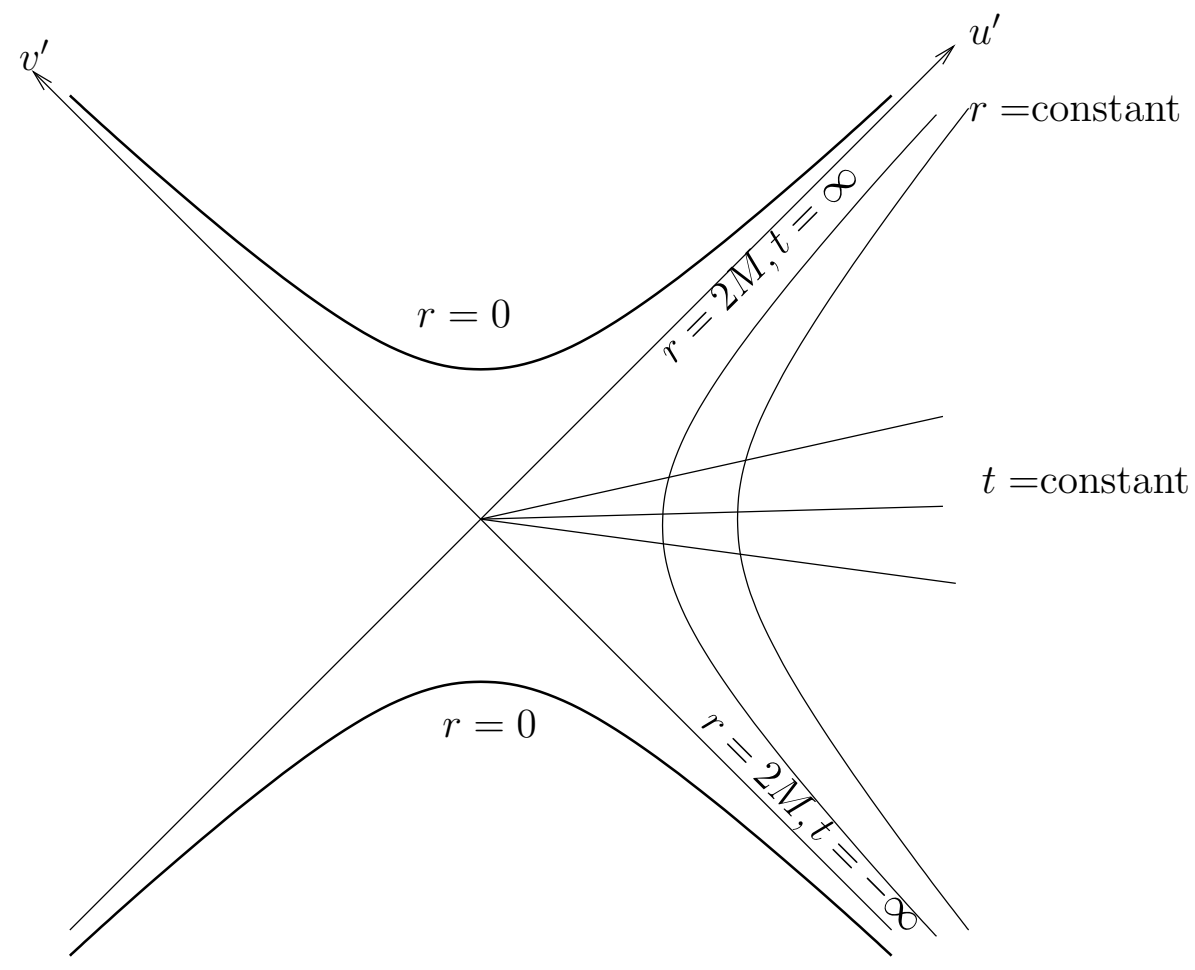

Figure 1: Kruskal diagram for Schwarzschild.

Another useful representation of the spacetime is the Penrose diagram [29, 30], which maps the whole spacetime to a finite region. Formally, we consider a manifold $(\bar{M}, \bar{g})$ such that the original spacetime $(M, g)$ maps to a subset of $\bar{M}$, with a conformal relation between the metrics, $\bar{g}_{\mu \nu}=\Omega^{2} g_{\mu \nu}$, on the image of $M$ in $\bar{M}$. The boundary of the image of $M$ in $\bar{M}$ is thought of as representing the 'points at infinity' in spacetime. This construction provides a highly useful tool for discussing the global structure of spacetimes. We can create an appropriate coordinate system describing $\bar{M}$ by setting

$$
\tan U=u^{\prime}, \quad \tan V=v^{\prime} .
$$


This is represented in figure 2. Once again, light cones lie at 45 degrees in the figure.

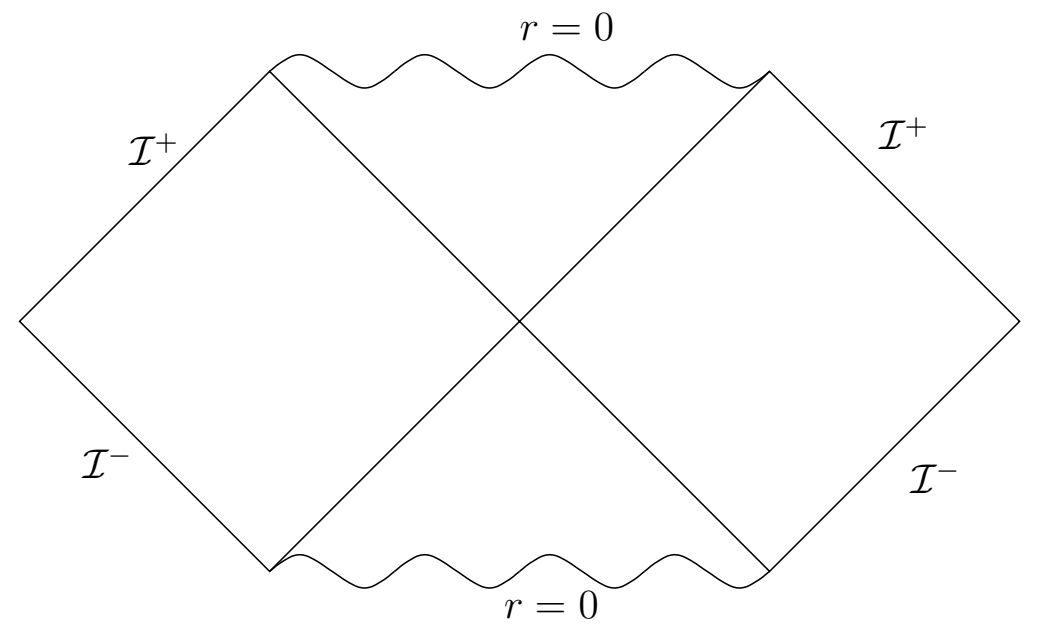

Figure 2: Penrose diagram for Schwarzschild.

This maximally-extended solution provides the model and template for our discussion of the properties of black holes and the relation to thermodynamics. We should note, however, that for a physical black hole formed by gravitational collapse, only a part of the spacetime is relevant, since this vacuum solution is only applicable outside of any collapsing matter. Furthermore, once we take Hawking radiation into account, a physical black hole will evaporate; the quantum-corrected black hole does not approach an equilibrium described by this solution. Thus, this eternal Schwarzschild solution is not directly related to the behaviour of real black holes.

This situation is somewhat improved if we consider a simple generalisation, to include a negative cosmological constant. Since this generalisation is of particular interest in string theory, we consider the solution in $d$ spacetime dimensions, the Schwarzschild-AdS metric

$$
d s^{2}=-f(r) d t^{2}+f(r)^{-1} d r^{2}+r^{2} d \Omega_{d-2},
$$

where

$$
\begin{aligned}
f(r) & =\left(1-\frac{w_{d} M}{r^{d-3}}+\frac{r^{2}}{\ell^{2}}\right), \\
w_{d} & =\frac{16 \pi G}{(d-2) \operatorname{Vol}\left(S^{d-2}\right)},
\end{aligned}
$$

$d \Omega_{d-2}$ is the metric on the unit $d-2$ sphere, and the cosmological constant is $\Lambda=$ $-(d-1) / \ell^{2}$. This has a coordinate singularity at $r=r_{+}$, where $f\left(r_{+}\right)=0$, which can be removed, as before, by passing to Kruskal coordinates. We define the light cone coordinates

$$
u, v=t \pm r_{*},
$$

where $d r_{*}=d r / f(r)$, and then define Kruskal coordinates by

$$
u^{\prime}=e^{\kappa u}, \quad v^{\prime}=-e^{-\kappa v},
$$


where

$$
\kappa=\frac{1}{2} f^{\prime}\left(r_{+}\right)=\frac{(d-1) r_{+}^{2}+(d-3) \ell^{2}}{2 r_{+} \ell^{2}} .
$$

The time-translation Killing vector is given by

$$
\partial_{t}=\kappa\left(u^{\prime} \partial_{u^{\prime}}-v^{\prime} \partial_{v^{\prime}}\right)
$$

In this case, $r_{*} \sim r^{-1}$ for large $r$, so $r_{*}$ has a finite range. This implies that the Kruskal diagram will have an additional boundary for some value of $u^{\prime} v^{\prime}$, as depicted in figure 3, corresponding to the timelike boundary in the asymptotically AdS spacetime.

In the Penrose diagram, this implies that the null asymptopia of the Schwarzschild solution is replaced by a timelike $\mathcal{I}$. It was recently noted [31] that for $d>3$, there is no choice of conformal factor such that both the singularity $r=0$ and the asymptotic boundaries $\mathcal{I}$ can be represented as straight lines on the Penrose diagram.
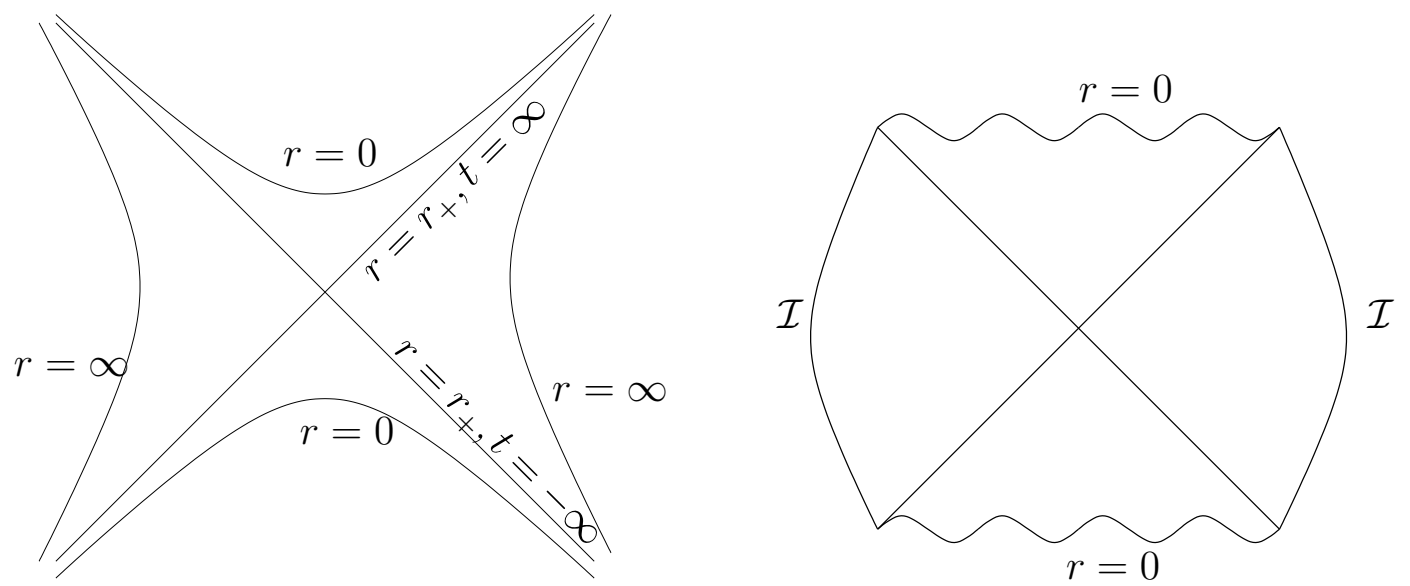

Figure 3: Kruskal and Penrose diagrams for Schwarzschild-AdS.

The study of the eternal black hole solution is a little more well-motivated in this case. The negative cosmological constant will prevent the radiation emitted from the black hole escaping to infinity. Furthermore, the specific heat is positive for large enough black holes (as we will see in section (5), so they can reach equilibrium with their Hawking radiation and settle down to a configuration approximately described by this eternal black hole solution.

The simple form of the above metrics is indicative of a genuine simplicity in the physics of black holes, and not just the special cases we have chosen to study. There are 'no-hair' theorems which state that Schwarzschild is the most general asymptotically flat static vacuum solution; this was first proved for the four-dimensional case in 32, and for higher dimensions in 33. In four dimensions, this generalises to stationary solutions: the most general stationary asymptotically flat black hole in four-dimensional vacuum general relativity is described by the Kerr solution, which has just two parameters: the mass $M$ and angular momentum $J$ (see [34] for a review of no-hair theorems). The most remarkable recent discovery in black hole physics is that no such no-hair theorem exists for stationary solutions in $d>4$ : the existence of 
black ring solutions 35 shows that there can be more than one solution with a given $M, J$.

\subsection{Horizons}

In the Schwarzschild-AdS solution, the surface at $r=r_{+}$is referred to as the black hole horizon or event horizon. It is actually an example of two different kinds of horizons. First, the surface $v^{\prime}=0$ is an event horizon for the right asymptotic region (and similarly $u^{\prime}=0$ is an event horizon for the left asymptotic region). An event horizon corresponds to the intuitive idea of a black hole as a region nothing can escape from: anything that crosses $v^{\prime}=0$ can never return. We can similarly define an event horizon in any spacetime with specified asymptotic behaviour: the event horizon $\mathcal{H}$ is the boundary of the causal past of the asymptotic infinity, $\mathcal{H}=\dot{J}^{-}\left(\mathcal{I}^{+}\right.$) (see [36] for a more detailed discussion). In a general spacetime, the region inside the event horizon which no signal can escape from, that is, the region $M-J^{-}\left(\mathcal{I}^{+}\right)$in a spacetime $M$, will be referred to as the black hole. By virtue of the definition, the event horizon in an arbitrary spacetime is always a null hypersurface. Generators may enter the horizon, but cannot leave it.

Because Schwarzschild-AdS is a static solution, the event horizon is also a Killing horizon. More accurately, the whole surface $r=r_{+}$, consisting of the two branches $u^{\prime}=0$ and $v^{\prime}=0$, forms a bifurcate Killing horizon. A Killing horizon is a null surface $\mathcal{N}$ whose generators are orbits of an isometry; that is, with a Killing vector field $\xi$ whose orbits generate $\mathcal{N}$. In Schwarzschild-AdS, both $u^{\prime}=0$ and $v^{\prime}=0$ are Killing horizons with respect to the time-translation Killing vector $\partial_{t}$.

A bifurcate Killing horizon is a structure like the space $r=r_{+}$: a pair of Killing horizons of the same Killing vector which intersect over a spacelike two-surface, called the bifurcation surface. The Killing vector $\xi$ necessarily vanishes on the bifurcation surface; that is, it is a collection of fixed points of the isometry. Conversely, if a Killing vector vanishes on a spacelike two-surface, there is a Killing horizon with that two-surface as a bifurcation surface [9]. Thus the bifurcate Killing horizon structure is entirely determined by these fixed points of the isometry. In Schwarzschild-AdS, the set $u^{\prime} v^{\prime}=0$ forms a bifurcate Killing horizon, and $u^{\prime}=v^{\prime}=0$ is the bifurcation two-sphere.

On a Killing horizon, the surface gravity $\kappa$ is defined by

$$
\xi^{\mu} \nabla_{\mu} \xi^{\nu}=\kappa \xi^{\nu} \quad \text { on } \mathcal{N}
$$

Equivalently,

$$
\kappa^{2}=-\frac{1}{2}\left(\nabla^{\mu} \xi^{\nu}\right)\left(\nabla_{\mu} \xi_{\nu}\right) \quad \text { on } \mathcal{N}
$$

In Schwarzschild, we can work out from (2.7) that $\kappa= \pm 1 / 4 M$. Similarly, in the Schwarzschild-AdS solution, $r=r_{+}$is a bifurcate Killing horizon with Killing vector $\xi=\partial_{t}$, and $\kappa=f^{\prime}\left(r_{+}\right) / 2$ is the surface gravity of this Killing horizon.

In general dynamical contexts, these two types of horizons are quite different. However, for the stationary black holes, they tend to coincide. That is, if the black 
hole solution has a Killing vector which is timelike at infinity, then in many cases of interest, the event horizon will also be a Killing horizon. This has been proved assuming that the solution is vacuum or electrovac [37, or if it has an additional angular symmetry, so that the black hole is stationary-axisymmetric [36].

The notion of an event horizon plays the central role in the general classical definition of a black hole, and in dynamical considerations such as the generalised second law. The Killing horizon structure will play the central role in the discussion of quantum field theory on this spacetime, and the derivation of black hole thermodynamics in section 5 will be based on the fact that the black hole horizon is a bifurcate Killing horizon. Thus, the Killing horizon structure is the more important for the purposes of these lectures. Note also that Killing horizons (unlike event horizons) do not occur only in black holes; there are many examples of Killing horizons which are not also black hole horizons. In fact, as we see next, this structure also occurs in flat space.

\section{$2.2 \quad$ Near-horizon structure}

We have argued above that the horizons in stationary black hole spacetimes will typically be bifurcate Killing horizons. Many of the universal features of black hole thermodynamics find their origin in this common structure, so we will now study the region of the spacetime near the horizon more closely. We focus on a neighbourhood of a null generator of the horizon in the Schwarzschild-AdS solution (the one at $\theta=0$, say) by considering

$$
r=r_{+}+\epsilon^{2} \frac{f^{\prime}\left(r_{+}\right)}{4} x^{2}
$$

for some small $\epsilon$, so $f(r)^{-1} d r^{2}=\epsilon^{2} d x^{2}$ up to corrections of order $\epsilon^{4}$, and also focus on small values of $\theta, \theta=\epsilon \rho$. In this limit the metric becomes

$$
d s^{2}=\epsilon^{2}\left(-\frac{f^{\prime}\left(r_{+}\right)^{2}}{4} x^{2} d t^{2}+d x^{2}+d s_{\mathbb{R}^{(d-2)}}^{2}\right)+O\left(\epsilon^{4}\right) .
$$

The leading part of the metric for small $\epsilon$ describes flat space (as we might expect, since we have focused on a small part of the spacetime), but not in the usual Cartesian coordinates. This coordinate system is called Rindler coordinates. If we set

$$
X=x \cosh (\kappa t), \quad T=x \sinh (\kappa t),
$$

where $\kappa=f^{\prime}\left(r_{+}\right) / 2$ as before, we recover flat space in the usual Cartesian coordinates,

$$
d s^{2}=\epsilon^{2}\left(-d T^{2}+d X^{2}+d s_{\mathbb{R}^{(d-2)}}^{2}\right) .
$$

The Rindler coordinates of (2.19) only cover the portion $X^{2}-T^{2}>0, X>0$ of flat space, which is called a Rindler wedge.

We see that there is a Killing horizon in flat space, as shown in figure 4. The surface at $x=0$ in the Rindler coordinates is a Killing horizon for the Killing vector $\xi=\partial_{t}$. This horizon is called a Rindler horizon. The Killing vector $\xi=\partial_{t}$ in the Rindler coordinates is recognised as a boost symmetry, and the co-moving observers 


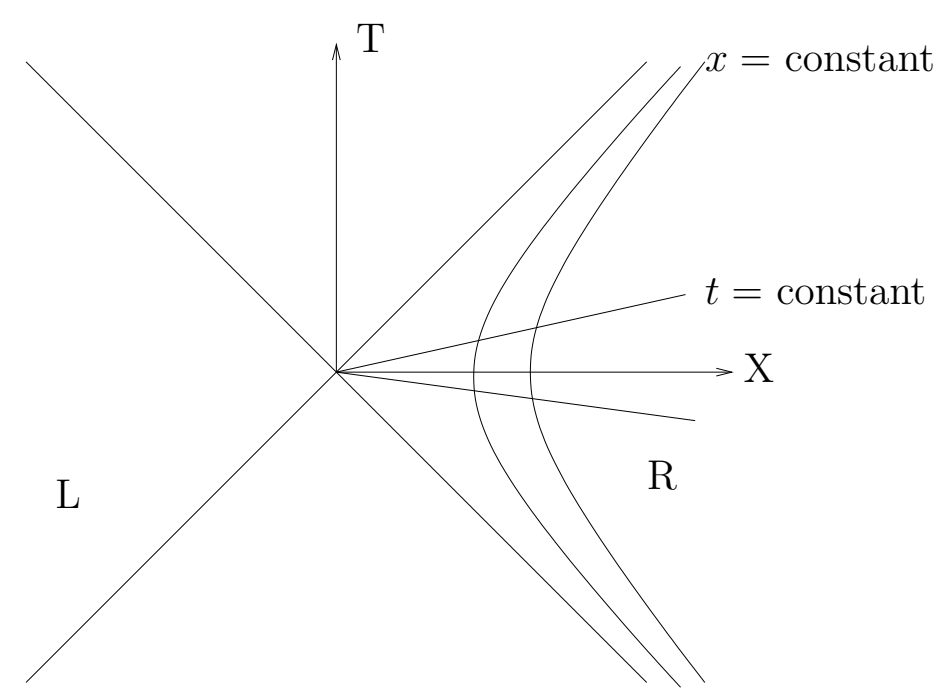

Figure 4: Rindler horizon in flat space.

in Rindler coordinates are uniformly accelerating, with acceleration $a=1 / x$. The horizon is therefore also referred to as an acceleration horizon. This horizon arises because these uniformly accelerating observers will not be able to receive signals from the whole spacetime. The Rindler observers, who follow worldlines $x=$ constant, cannot influence or be influenced by events in the left wedge in figure 4 . Of course, since this is just flat space, this is not an event horizon; it is an example of an observerdependent horizon, specified by reference to a particular observer's worldline.

The Rindler horizon is the simplest example of a Killing horizon, and provides a template for any investigation of Killing horizons. We can extend the above analysis to show that the near-horizon limit of an arbitrary bifurcate Killing horizon is a Rindler horizon. The fact that we can in this sense reduce the local properties of any bifurcate Killing horizon to considering a Rindler horizon will play a central role in the universality of the thermodynamic properties of black holes.

\section{Classical black hole thermodynamics}

In this lecture, we will discuss the features of classical black hole mechanics which provided the first indications that there was a relation between black holes and thermodynamics. We aim to give a qualitative description, providing a flavour for the results and a few illustrative examples. For details of proofs and fuller arguments, we refer the reader to the references; the lecture notes on black holes by Townsend 38] include proofs of many of the statements we quote here.

\subsection{Area theorem}

We consider first Hawking's area theorem [39] and the relation to the second law of thermodynamics. The area theorem states: If the spacetime on and outside the future event horizon is a regular predictable space, and the stress tensor satisfies the 
null energy condition, $T_{\mu \nu} k^{\mu} k^{\nu} \geq 0$ for arbitrary null $k^{\mu}$, then the area of spatial crosssections of the event horizon is non-decreasing. The condition that the spacetime is a regular predictable space essentially forbids naked singularities on or outside the event horizon; see [36] for details. Thus, so long as spacetime is regular, and the matter satisfies an energy condition, the area of the event horizon is non-decreasing. Bekenstein pointed out [40] that there was a close analogy between this result and the second law of thermodynamics, and used it and thermodynamic considerations to argue that black holes should be assigned an entropy proportional to the area of the event horizon.

Let us briefly give an idea of how this theorem is proved. If we consider a small bundle of the null geodesics generating the event horizon, which has a cross-sectional area $A$ at some value of the affine parameter $\lambda$ along the geodesics, then we can define the expansion $\theta$ by

$$
\frac{d A}{d \lambda}=\theta A
$$

that is, $\theta$ is the fractional rate of change of the area. If we imagine the theorem is violated, so that the area of the horizon decreases, then we must have $\theta<0$ somewhere on the event horizon. Since the generators are geodesics, the evolution of the expansion is determined by Raychaudhuri's equation,

$$
\frac{d \theta}{d \lambda}=-\frac{1}{3} \theta^{2}-\sigma_{\mu \nu} \sigma^{\mu \nu}+\omega_{\mu \nu} \omega^{\mu \nu}-R_{\mu \nu} k^{\mu} k^{\nu}
$$

where $\sigma_{\mu \nu}$ is the shear, $\omega_{\mu \nu}$ is the rotation, which vanishes for the generators of a hypersurface, and $k^{\mu}$ is the tangent to the null geodesics. Hence, if the null energy condition is satisfied, so $R_{\mu \nu} k^{\mu} k^{\nu} \geq 0, \theta<0$ implies that $\theta \rightarrow-\infty$ in finite $\lambda$. This produces a caustic, as shown in figure 5. But the points $p$ and $q$ indicated on the figure are timelike separated. This contradicts our assumption that the null curves are the generators of an event horizon, as no two points on the event horizon can be timelike separated. Thus, by contradiction, the cross-sectional area of an event horizon cannot decrease. It is interesting to note that although the proof assumes Einstein's equations, they are not used in an essential way, whereas they will be in proving the first law. The assumption that the geometry is regular on and outside the event horizon will continue to play an important role throughout our discussion.

\subsection{Zeroth and first laws}

The area theorem is the only one of the classical results which truly concerns the dynamics of black hole event horizons. The zeroth and first laws of black hole mechanics are concerned with equilibrium or quasi-equilibrium processes. That is, they concern stationary black holes, or adiabatic changes from one stationary black hole to another. We will assume that for such black holes, the event horizon is also a Killing horizon, using one of the arguments at the end of section 2.1 - this implies some limitations on the generality of the following statements.

The zeroth law of black hole mechanics then states that the surface gravity $\kappa$ is constant over the event horizon of a stationary black hole [41]. We have already 


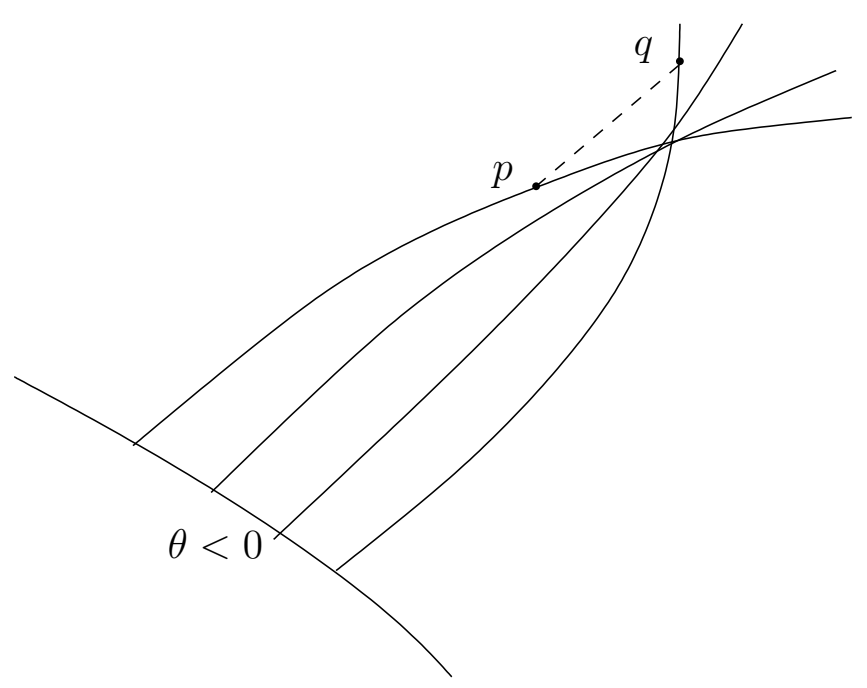

Figure 5: A family of null geodesics with $\theta<0$ initially will form a caustic; the dotted curve connecting $p$ and $q$ lies within the local light cone, so these points are timelike separated.

seen that this is true for Schwarzschild and Schwarzschild-AdS. This provides a first indication that the surface gravity is an analogue of the temperature. This may seem a weak analogy, since there are presumably many constant quantities in a stationary black hole solution. Nonetheless, this is a non-trivial statement. If we consider for example the non-uniform black string solution which was discovered in [42, whose horizon is depicted in figure 6. many local features of the event horizon, such as its local radius of curvature, vary over the horizon, but the surface gravity is constant by virtue of the above result.

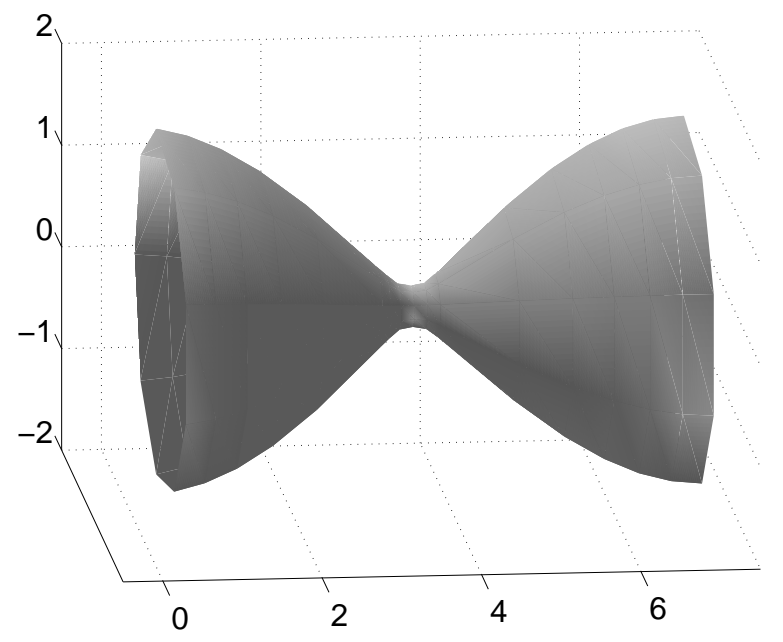

Figure 6: The event horizon of a non-uniform black string solution, from 42].

The analogy is considerably strengthened by the first law of black hole mechanics. 
For a rotating charged black hole, this states that

$$
d M=\frac{\kappa}{8 \pi} d A+\Omega d J+\Phi d Q
$$

where $\Omega$ is the angular velocity and $\Phi$ is the electric potential [41]. This relates the change in area to the change in mass, angular momentum and charges for an adiabatic transition between nearby stationary black hole solutions. This first law is evidently analogous to the first law of thermodynamics. Taken together with the second law of thermodynamics, which identifies a multiple of the area as the analogue of entropy, it enables us to identify a multiple of the surface gravity $\kappa$ as an analogue of temperature. Later, we reverse this argument: we can identify $T=\frac{\hbar \kappa}{2 \pi}$ as the temperature of a black hole using quantum field theory in curved spacetime. The first law then implies that $S=\frac{A}{4 G \hbar}$ is the black hole's entropy.

If we consider for example the Schwarzschild family of solutions, it is straightforward to prove (3.3) by direct calculation: the area of the event horizon is $A=16 \pi M^{2}$, so under a small change $M \rightarrow M+d M, A \rightarrow 16 \pi(M+d M)^{2} \approx A+32 \pi M d M$. Recalling that $\kappa=1 / 4 M$, we see that indeed $d M=\kappa d A / 8 \pi$.

Similarly, if we consider the Schwarzschild-AdS black hole, for which

$$
A=\operatorname{Vol}\left(S^{d-2}\right) r_{+}^{d-2}
$$

then

$$
d A=\operatorname{Vol}\left(S^{d-2}\right)(d-2) r_{+}^{d-3} d r_{+},
$$

and we can relate $d r_{+}$to $d M$ by observing that

$$
\begin{aligned}
0 & =f_{M+d M}\left(r_{+}+d r_{+}\right)=f_{M+d M}\left(r_{+}\right)+d r_{+} f_{M}^{\prime}\left(r_{+}\right) \\
& =\left(1-\frac{\omega_{d}(M+d M)}{r_{+}^{d-3}}+\frac{r_{+}^{2}}{l^{2}}\right)+d r_{+} f_{M}^{\prime}\left(r_{+}\right) \\
& =-\frac{\omega_{d} d M}{r_{+}^{d-3}}+d r_{+} f_{M}^{\prime}\left(r_{+}\right)
\end{aligned}
$$

Hence,

$$
d M=\frac{r_{+}^{d-3} f^{\prime}\left(r_{+}\right)}{\omega_{d}} d r_{+}=\frac{f^{\prime}\left(r_{+}\right)}{16 \pi}(d-2) \operatorname{Vol}\left(S^{d-2}\right) r_{+}^{d-3} d r_{+}=\frac{\kappa}{8 \pi} d A
$$

where we have used $\omega_{d}=16 \pi /(d-2) \operatorname{Vol}\left(S^{d-2}\right)$ and $\kappa=f^{\prime}\left(r_{+}\right) / 2$. Both these demonstrations have used the equations of motion implicitly, as we have used details of the form of the solutions.

For more general asymptotically flat rotating charged black holes, it is convenient to prove the first law by using the Komar integrals to define $M, J$, and $Q$. As with the short proof above for the Schwarzschild and Schwarzschild-AdS cases, the proof using Komar integrals uses the equations of motion in an essential way.

Since $M, J, Q$ are defined asymptotically, the form of the first law derived in this way is relating quantities defined at infinity to quantities defined locally on the black 
hole horizon. This seems to depend in a crucial way on the black hole uniqueness results, which ensure that there is a unique spacetime geometry, and hence a welldefined value of $A$, for given $M, J, Q$. In higher dimensions, the solutions are no longer unique [35, and can involve 'dipole charges' which are not globally conserved, and hence are not given by asymptotic integral expressions [43. It was shown in [43] that these latter solutions satisfied a first law including a work term associated with the dipole charge. Understanding the first law for these cases in general is an interesting open problem.

A potentially relevant development is the recent definition of a form of the first law which is valid locally on the horizon, by introducing a new notion of a horizon called an isolated horizon 44. This also allows discussion of thermodynamics of black holes assuming that only the black hole horizon is in equilibrium, not necessarily the whole spacetime.

Another important generalisation, which has already played an important role in string theory discussions, is the investigation by Wald and Iyer [45, 46] (building on ideas from [47, 48]), who showed that a first law holds for an arbitrary Lagrangian theory of gravity, where the gravitational Lagrangian $\mathbf{L}\left(R_{\mu \nu \rho \sigma}, \nabla_{\lambda} R_{\mu \nu \rho \sigma}, \ldots\right)$ is some general functional of the Riemann tensor and its covariant derivatives - this could be, for example, the effective action for a string theory including $\alpha^{\prime}$ corrections.

This approach uses a Noether current $\mathbf{J}$ associated with a vector field $\xi$ constructed from a diffeomorphism variation of the Lagrangian. When the equations of motion are satisfied, this current defines an $(n-2)$ form charge $\mathbf{Q}[\xi]$ on spacetime satisfying $\mathbf{J}=d \mathbf{Q}$. They showed that if one considered a stationary black hole solution in this theory, with a bifurcate Killing horizon $\mathcal{H}$, one could relate an energy $\mathcal{E}$ defined by a surface integral at infinity to the change in a local integral over the horizon,

$$
\delta \mathcal{E}=\frac{\kappa}{2 \pi} \delta S+\text { work terms, }
$$

where $\kappa$ is the surface gravity defined as before, and the quantity playing the role of entropy is now

$$
S=2 \pi \int_{\Sigma} \mathbf{Q}[\bar{\xi}]=2 \pi \int_{\Sigma} \mathbf{X}^{c d} \epsilon_{c d}
$$

In the expression for $S, \Sigma$ is the bifurcation surface in the bifurcate Killing horizon $\mathcal{H}$, and $\bar{\xi}$ is the Killing vector field which vanishes on this bifurcation surface. The second form of the expression is obtained from the explicit form of the Noether charge. The $n-2$ form $\mathbf{X}$ can be defined by taking a functional derivative of the Lagrangian with respect to the Riemann tensor,

$$
\mathbf{X}_{a b}=-\frac{\delta \mathbf{L}}{\delta \mathbf{R}_{a b}}
$$

where $\mathbf{R}_{a b}$ is the usual curvature 2-form, $R_{a b \mu \nu}=e_{a}^{\lambda} e_{b}^{\rho} R_{\lambda \rho \mu \nu}$.

This construction of the first law provides a notion of entropy for a general gravitational theory. If the Lagrangian is simply the Einstein-Hilbert Lagrangian, the form $\mathbf{X}_{a b}$ will be proportional to the area element on the horizon, and this will reduce 
to $S=A / 4$. In general, the entropy is given by a local, geometrical integral over the event horizon of the black hole, but this integral may now involve the curvature as well as the proper area of the horizon. Since (3.8) is proved for a general perturbation in the space of solutions, this formulation may also be sufficiently general to encompass the first law for black rings with dipole charges.

This formula for the entropy has been applied to the calculation of the entropy for extremal black holes in string theory, including higher-curvature corrections [49, 50, 51. ${ }^{2}$ Remarkably, the changes in the entropy formula agree precisely with the results of microscopic calculations from an M-theoretic perspective [52, 53]. More recently, it has been suggested that the partition function for these black holes, including all the higher-curvature corrections, is related to the partition function for topological strings [54.

An obvious next question is whether this entropy satisfies a second law; that is, can one show that (3.9) is always non-decreasing in arbitrary physical processes, proving an analogue of Hawking's area theorem for this more general expression? For quasistationary processes, the second law can be shown to hold as a consequence of the first law and the null energy condition [55]; however, for general dynamical processes, the question remains open, although a proof for a particular class of Lagrangians was given in [55].

Already in this generalisation of the classical theory, we see that the form of the temperature is unchanged, while the form of the entropy appearing in this formula depends on the theory we consider. The primary nature of the identification of the temperature with surface gravity in (1.1) will become still more apparent when we consider quantum effects in the next two sections.

In this section, we have seen that there is an analogy between the classical laws of black hole mechanics and the laws of thermodynamics. However, at the classical level, this is purely an analogy - in particular, classical black holes are not thermal, as they have no emission. To promote this analogy to a true identification, we need to introduce a quantum element into our discussion, by studying quantum field theory on the black hole background.

\section{Quantum field theory in curved spacetime}

The subject of quantisation of the matter fields on a fixed spacetime background is of clear practical importance. It provides the quantum analogue of the classical kinematics of gravity determined by for example the geodesic equation, and like the geodesic equation, it should provide a good description in contexts where we are interested in the effects of spacetime on some matter, but the gravitational field of this matter itself is negligible. It is also analogous to quantising the fields describing charged particles in a fixed classical background electromagnetic field.

\footnotetext{
${ }^{2}$ These extremal black holes have $T=0$, and their horizons are not bifurcate Killing horizons. To apply the formula (3.9) to calculate their entropy, we must assume that the entropy is continuous as we move from the non-extreme black holes, where the argument of [45] applies, to these extreme solutions.
} 
Unlike quantisation in a fixed background gauge field, quantisation on curved spacetime involves significant conceptual differences from the more familiar case of quantisation in flat space. This is because in the usual treatment of quantum field theory, Poincare-invariance plays a central role in the discussion. In considering a general spacetime background, we have to give up on this approach based on global symmetry. This means in particular that there will not be a preferred vacuum state or a natural Fock space construction of the Hilbert space. We are led to make a strong distinction between the quantisation of the field, promoting it to a local operator, and issues to do with the choice of state.

In this section, I will briefly introduce the essential aspects of this quantisation. The initial review of canonical quantisation will follow the treatment in [9, 28, quite closely, adopting a modern point of view where the focus is on promoting fields to local operators and the invariant characterisation of states, rather than mode decompositions and Fock spaces. I will also briefly discuss the Peierls bracket, which provides an alternative, more covariant approach to quantisation. Since our focus is on thermodynamics, I will spend some time explaining the KMS condition, which provides a characterisation of thermal states for general quantum systems, and its application to a quantum field on a stationary background spacetime.

Our discussion will be restricted to free theory, and it will be sufficient for our purposes to consider a real scalar field. The extension to other spins is fairly straightforward. So we start from a classical theory with action

$$
S=-\frac{1}{2} \int d^{d} x \sqrt{-g}\left(g^{\mu \nu} \nabla_{\mu} \varphi \nabla_{\nu} \varphi+m^{2} \varphi^{2}\right),
$$

with corresponding equation of motion

$$
\left(\square-m^{2}\right) \varphi=0 .
$$

\subsection{Canonical quantisation}

If we assume our spacetime manifold $M$ can be decomposed as $M=\mathbb{R} \times \Sigma$ where $\mathbb{R}$ represents the timelike direction and $\Sigma$ is some $d-1$-dimensional Riemannian manifold, then we can apply the usual techniques of canonical quantisation. Denoting the corresponding decomposition of a coordinate chart on $M$ by $x^{\mu}=\left(x^{0}, \vec{x}\right)$, we can write

$$
S=\int d x^{0} L
$$

and introduce as canonical coordinates the value of $\varphi$ on $\Sigma$ at some value of $x^{0}, \varphi(\vec{x})$, and the conjugate momentum

$$
\pi(\vec{x})=\frac{\delta L}{\delta\left(\partial_{0} \varphi\right)}=\sqrt{-g} g^{\mu 0} \partial_{\mu} \varphi=-\sqrt{h} n^{\mu} \partial_{\mu} \varphi(\vec{x}),
$$

where $h_{i j}$ is the metric on $\Sigma$ and $n_{\mu}$ is the normal to $\Sigma$ in $M$.

We then quantise by imposing the usual canonical commutation relations,

$$
[\varphi(\vec{x}), \pi(\vec{x})]=i \hbar \delta^{d-1}(\vec{x}, \vec{y}),
$$


where the densitized delta function $\delta^{d-1}(\vec{x}, \vec{y})$ is defined by

$$
\int_{\Sigma} d^{d-1} \vec{x} f(\vec{y}) \delta^{d-1}(\vec{x}, \vec{y})=f(\vec{x})
$$

This is the essence of quantisation. It is worth noting that this step is entirely local. The overall structure of the spacetime does not enter into the definition of these commutation relations. It is only when we wish to go on to represent them in terms of operators acting on a Hilbert space that the structure of the background becomes important, entering notably through the definition of the inner product.

The usual flat-space definition of this Klein-Gordon inner product generalises directly to curved spacetime,

$$
(f, g)=\int_{\Sigma} d \Sigma_{\mu} j^{\mu}(f, g)
$$

where the current

$$
j^{\mu}(f, g)=-i \sqrt{-g} g^{\mu \nu}\left(\bar{f} \partial_{\nu} g-\partial_{\nu} \bar{f} g\right) .
$$

This current is conserved as a consequence of the equations of motion, $\partial_{\mu} j^{\mu}=0$, which implies that the inner product $(f, g)$ is independent of the choice of spacelike slice $\Sigma$; that is, it is in particular independent of the time coordinate $x^{0}$. As a consequence of the definition, this inner product also satisfies

$$
\overline{(f, g)}=-(\bar{f}, \bar{g})=(g, f)
$$

and as a result, $(f, \bar{f})=0$. The inner product is clearly not positive definite.

So far, the quantisation procedure has precisely parallelled the usual discussion. At this stage in flat space, we would introduce a basis of positive frequency field modes. The restriction of the inner product (4.7) to these modes would then be positive definite, and we could use it to construct the Hilbert space. The essential difference in a curved spacetime is that there is no natural a priori notion of positive frequency. To construct a Hilbert space, we therefore need to introduce a decomposition of the space $\mathcal{S}$ of solutions of the field equations into a positive norm part and its conjugate:

$$
\mathcal{S}=\mathcal{S}_{p} \oplus \overline{\mathcal{S}}_{p}
$$

where

$$
(f, f)>0 \quad \forall f \in \mathcal{S}_{p}, \quad(f, \bar{g})=0 \quad \forall f, g \in \mathcal{S}_{p} .
$$

Given such a choice of decomposition, we can define annihilation and creation operators for the mode $f$ by

$$
a(f)=(f, \varphi), \quad a^{\dagger}(f)=-a(\bar{f})=-(\bar{f}, \varphi) .
$$

The canonical commutation relations imply that these operators will satisfy the usual algebra of creation and annihilation operators,

$$
\left[a(f), a^{\dagger}(g)\right]=(f, g), \quad[a(f), a(g)]=\left[a^{\dagger}(f), a^{\dagger}(g)\right]=0 .
$$


We may, if we wish, identify a vacuum state associated with this decomposition of the space of solutions by

$$
a(f)|0\rangle=0 \quad \forall f \in \mathcal{S}_{p},
$$

and construct a Fock space by taking the span of all states of the form

$$
a^{\dagger}\left(f_{i_{1}}\right) \ldots a^{\dagger}\left(f_{i_{n}}\right)|0\rangle
$$

for all $n$ and all $f_{i_{k}} \in \mathcal{S}_{p}$.

Given a choice of positive norm subspace $\mathcal{S}_{p}$, we can construct an orthonormal basis $f_{n} \in \mathcal{S}_{p}$. Writing the annihilation and creation operators associated to this basis as $a_{n}=\left(f_{n}, \varphi\right), a_{n}^{\dagger}=-\left(\bar{f}_{n}, \varphi\right)$, we can then make contact with the standard treatment of quantisation by introducing the usual mode decomposition of the field operator

$$
\varphi=\sum_{n}\left(a_{n} f_{n}+a_{n}^{\dagger} \bar{f}_{n}\right)
$$

and the usual $n$-particle basis of states for the Fock space,

$$
a_{n_{1}}^{\dagger} \ldots a_{n_{k}}^{\dagger}|0\rangle \text {. }
$$

The essential difficulty in this construction is the specification of a positive norm subspace $\mathcal{S}_{p} \subset \mathcal{S}$, which corresponds physically to the specification of a notion of positive frequency. It is always possible to find such subspaces, but in a general curved spacetime the background structure of the spacetime does not select any particular one as a natural candidate for defining the vacuum and Fock space. It can be shown [9] that the Fock space constructions based on different notions of positive frequency will be unitarily inequivalent. That is, different constructions are giving genuinely different representations of the algebra of field operators, and the vacuum constructed with respect to one notion of positive frequency $\mathcal{S}_{p},|0\rangle_{p}$, will not lie in the Fock space built on the vacuum $|0\rangle_{p^{\prime}}$ associated with a different choice $\mathcal{S}_{p^{\prime}}$.

There is a linear relation between different choices: any $f^{\prime} \in \mathcal{S}_{p^{\prime}}$ can be written as $f^{\prime}=f+\bar{g}$ for some $f, g \in \mathcal{S}_{p}$. If we introduce bases, this linear relation can be expressed through the Bogoliubov transformation

$$
f_{n}^{\prime}=\sum_{m} \alpha_{n m} f_{m}+\beta_{n m} \bar{f}_{m}
$$

The mixing of positive and negative frequency is expressed through the presence of the coefficients $\beta_{n m}$, which could not appear in the transformation between different bases for the same $\mathcal{S}_{p}$.

\subsection{Peierls bracket}

The use of a canonical decomposition of spacetime, splitting it into a Riemannian spatial manifold $\Sigma$ and a time direction, appears contrary to the spirit of special and general relativity. As Minkowski presciently observed, "space by itself, and time by itself, are doomed to fade away into mere shadows and only a kind of union of the two 
will retain an independent reality" [56. While it has been shown that the canonical quantisation described above is independent of the particular foliation of spacetime we choose, it would be preferable to have a covariant method which avoided the need to introduce a foliation altogether.

Fortunately, such a method exists. It is due to Peierls [57]. The key idea of the method is to introduce a bracket between classical observables which is constructed entirely covariantly, but which is equivalent to the Poisson bracket of the conventional canonical treatment. The Peierls bracket formalism and covariant quantisation is described in great detail and generality in [23. There is also a useful brief discussion in 21 .

Consider classical observables $A, B$, which can be integrals of some arbitrary local functions of the fields and derivatives over a region of spacetime. To construct their Peierls bracket, consider adding an infinitesimal contribution to the Lagrangian

$$
\mathcal{L} \rightarrow \mathcal{L}+\epsilon A
$$

This will produce a corresponding change in the solutions of the equations of motion: we consider the change with advanced or retarded boundary conditions $\delta_{A}^{ \pm} \varphi$, which vanish respectively in the region to the future or past of the perturbation $A$. These define corresponding changes $\delta_{A}^{ \pm} B$ in the other observable $B$.

The difference between the advanced and retarded solutions,

$$
\delta_{A} \varphi=\delta_{A}^{-} \varphi-\delta_{A}^{+} \varphi
$$

defines an action of $A$ on the space of classical solutions, as $\varphi+\epsilon \delta_{A} \varphi$ satisfies the equations of motion of the original Lagrangian $\mathcal{L}$ to first order in $\epsilon$. The Peierls bracket is then defined as the action of $A$ on $B$ :

$$
(A, B) \equiv \delta_{A} B=\delta_{A}^{-} B-\delta_{A}^{+} B .
$$

There is also a natural reciprocity relation, $\delta_{A} B=-\delta_{B} A$, which implies that this can also be expressed as the action of $B$ on $A$. Note that because $\delta_{A} \varphi$ defined a motion in the space of classical solutions, the Peierls bracket is only defined on-shell; that is, (4.21) only defines a bracket between the observables $A, B$ evaluated on some classical solution.

In our example of a free real scalar field, the Peierls bracket of the fundamental fields is

$$
\left(\varphi(x), \varphi\left(x^{\prime}\right)\right)=i \Delta\left(x-x^{\prime}\right),
$$

where $\Delta\left(x-x^{\prime}\right)$ is the difference between the retarded and advanced propagators. This is the same as the Poisson bracket of the fields, establishing for this particular case the general equivalence of the Peierls bracket and the Poisson bracket. We can quantise the theory covariantly by simply promoting the fields to operators and imposing the Peierls bracket as a commutator relation on the field operators:

$$
\left[\varphi(x), \varphi\left(x^{\prime}\right)\right]=i \Delta\left(x-x^{\prime}\right) .
$$

This produces the same algebra of observables as the canonical quantisation while avoiding the need to introduce any canonical structures. 


\subsection{Observables \& Hadamard states}

We distinguish different states in quantum field theory by the expectation values they give for certain operators. In flat spacetime, states are labelled with the value of the particle number operator $N=\sum_{n} a_{n}^{\dagger} a_{n}$. Intuitively, states with larger $\langle N\rangle$ are 'farther' from the vacuum. In a general curved spacetime, however, where there is no natural notion of positive frequency, this value depends on an arbitrary choice we are making when we define $a_{n}$, so it cannot be a physically meaningful quantity. There is no invariant notion of the number of particles in the space, even at a fixed moment in time.

We therefore want to label states not by particle number, but with the values for physical observables. A class of simple operators we can consider are the time-ordered products of the fundamental field operator. The expectation values of these operators are sufficient to distinguish between different states. That is, we consider the $n$-point expectation values of fundamental field operators, $\langle\varphi(x) \varphi(y)\rangle,\langle\varphi(x) \varphi(y) \varphi(z)\rangle$, etc. In our particular case of a free scalar field, a state can be specified by specifying the two-point function, ${ }^{3}$

$$
G(x, y)=\langle\varphi(x) \varphi(y)\rangle .
$$

However, there are quantities we are physically interested in which are not the expectation value of any operator of this form. For gravitational purposes, the most important example is the stress-energy tensor $\left\langle T_{\mu \nu}\right\rangle$, the source of the gravitational field in a semi-classical treatment. The classical stress-energy tensor cannot be simply promoted to a quantum operator, because it involves the product of fields at the same spacetime point, and these products are not well-defined for the distributional field operators of the quantum theory.

In flat spacetime, we deal with this problem by normal ordering, rewriting the stress tensor in terms of the annihilation and creation operators $a_{n}, a_{n}^{\dagger}$ (whose product is well-defined), with a particular choice of operator ordering, which corresponds to measuring the difference between $\left\langle T_{\mu \nu}\right\rangle$ in the state of interest and its value in the vacuum. However, once again, such an expression in terms of creation and annihilation operators will not produce invariant results in curved spacetimes.

The question is then, is there some alternative approach to the construction of $\left\langle T_{\mu \nu}\right\rangle$ ? Remarkably, it has been shown (see 9] for details) that one can construct a local, conserved stress-energy tensor $\left\langle T_{\mu \nu}\right\rangle$, but only for a certain class of states, called Hadamard states.

Hadamard states are defined by the requirement that at short distances, the twopoint function behave as

$$
\lim _{x \rightarrow y} G(x, y)=\frac{U(x, y)}{4 \pi^{2} \sigma(x, y)}+V(x, y) \ln \sigma+W(x, y),
$$

where $\sigma$ is half the square of the geodesic distance between $x$ and $y$, and $U, V$, and $W$ are smooth functions with $U(x, x)=1$. The form of the functions $U(x, y)$

\footnotetext{
${ }^{3}$ The states where the one-point function vanishes and higher-point functions are determined by the two-point function are termed quasi-free 9. We will assume henceforth that the state is quasi-free, so that the two-point function suffices to uniquely specify the state.
} 
and $V(x, y)$ is then determined by requiring that the two-point function satisfies the Klein-Gordon equation in $x$. This restriction on the form of the two-point function can be motivated by observing that it is satisfied by the two-point function in any state in the Fock space built on the usual Minkowski vacuum in flat space. Thus, the restriction to Hadamard states corresponds to the reasonable assumption that when we consider the behaviour on scales much smaller than any curvature scale, using our usual flat-space techniques will be a good approximation.

Thus, the short-distance singularity in Hadamard states is entirely determined by the local spacetime geometry. They are the closest we can come in a curved spacetime to our usual Fock space states built on the Minkowski vacuum: the constraint on the short distance singularities will mean that all physical observers will see a finite density of particles in any Hadamard state. We can remove this ultraviolet divergence by a local and state-independent renormalisation procedure. The renormalised two-point function can be used to construct $\left\langle T_{\mu \nu}\right\rangle$, giving a unique result up to the addition of local curvature terms (which represent a renormalisation of the gravitational couplings) 9 .

The Hadamard condition is preserved by evolution, so if the state is initially Hadamard in a suitable neighbourhood of some Cauchy surface, it will be Hadamard throughout the spacetime [58. It has also been shown that if the spacetime is spatially compact, any Hadamard state can be constructed as a state in the Fock space defined on any other Hadamard state [9]. These considerations all motivate adopting the Hadamard condition as a natural condition on states.

\subsection{KMS condition}

In this subsection, we will finally describe the general characterisation of thermal states in quantum field theory; this is provided by the KMS condition, named for Kubo [59] and Martin and Schwinger 60]. We first give a brief description of the KMS condition, motivating it by relating it to the usual notion of thermal equilibrium for a system with finitely many degrees of freedom. We then discuss the application of this condition to a free scalar field, and show that thermal states of a free scalar field are characterised by having a two-point function which is 'periodic in imaginary time'. More detailed discussions of the KMS condition can be found in [21, 61. My discussion of the KMS condition for a scalar field follows 8 .

In a system with finitely many degrees of freedom, the standard definition of the canonical ensemble describing thermal equilibrium at temperature $T=\beta^{-1}$ is that for all operators $A$, the expectation value is given by

$$
\langle A\rangle_{\beta}=\frac{1}{Z} \operatorname{Tr}\left(e^{-\beta H} A\right)
$$

where $H$ is the Hamiltonian of the system and $Z=\operatorname{Tr}\left(e^{-\beta H}\right)$ is the canonical partition function. However, for a system with a continuous spectrum, such as a field theory in infinite volume, this can only provide a formal definition of thermal behaviour, since this partition function diverges (as will $\operatorname{Tr}\left(e^{-\beta H} A\right)$ for most operators of interest). 
A more general characterisation of thermality can be obtained by considering the consequences of the above definition. Consider the expectation value

$$
\left\langle A_{t} B\right\rangle=\left\langle e^{i t H} A e^{-i t H} B\right\rangle
$$

and regarding this as a function of time, extend the index $t$ to imaginary values. Then, in the case of a simple quantum mechanical system, we can deduce from (4.26) that

$$
\left\langle A_{-i \beta} B\right\rangle_{\beta}=\frac{1}{Z} \operatorname{Tr}\left[e^{-\beta H}\left(e^{\beta H} A e^{-\beta H}\right) B\right]=\frac{1}{Z} \operatorname{Tr}\left[e^{-\beta H} B A\right]=\langle B A\rangle_{\beta}
$$

for all bounded operators $A, B$, where we have used the cyclic property of the trace in the second step. This provides us with a condition that only makes reference to finite quantities, the KMS condition [59, 60]

$$
\left\langle A_{-i \beta} B\right\rangle_{\beta}=\langle B A\rangle_{\beta}
$$

Any state such that (4.29) holds for all bounded operators $A, B$ is called a KMS state.

This condition has been derived as a consequence of (4.26), but it provides a suitable notion of thermal states for more general systems. It has been shown to be equivalent to (4.26) where that definition applies. Furthermore, if an infinite system satisfies the KMS condition, any finite system coupled to it will approach thermal equilibrium in the sense of (4.26); thus, a system satisfying (4.29) behaves as a thermal reservoir. Finally, a system satisfying the KMS condition minimises the free energy locally. For proofs of these statements, see [21, 61.

We will now specialise to the case of a free scalar field in curved spacetime, and investigate the application of the KMS condition, following [8] quite closely. We observe first that an equilibrium state is by definition a stationary state, so for any notion of equilibrium state to exist, the spacetime must admit a timelike Killing vector field, with associated observers whose worldlines are orbits of the Killing vector field; the notion of equilibrium will be with respect to measurements by these observers. Call this Killing vector $\partial_{t}$.

The observable of interest in the theory of a free scalar field is the two-point function $\langle\varphi(x) \varphi(y)\rangle$; the Killing symmetry implies that this only depends on the time difference, $t_{x}-t_{y}$. We introduce the positive and negative frequency Wightman functions

$$
G_{+}\left(t_{x}-t_{y}, \vec{x}, \vec{y}\right)=\langle\varphi(x) \varphi(y)\rangle
$$

and

$$
G_{-}\left(t_{x}-t_{y}, \vec{x}, \vec{y}\right)=\langle\varphi(y) \varphi(x)\rangle=G_{+}\left(t_{x}-t_{y}, \vec{x}, \vec{y}\right)+[\varphi(x), \varphi(y)]
$$

where the $c$-number commutator is the same for all states.

Extending the dependence on $t_{x}-t_{y}$ into the complex plane, one can show that $G_{+}^{\beta}(z, \vec{x}, \vec{y})$ is holomorphic below the real axis, for $-\beta<\Im(z)<0$, while $G_{-}^{\beta}(z, \vec{x}, \vec{y})$ is holomorphic for $0<\Im(z)<\beta$, where the superscript implies that we take the 
expectation values on the RHS of (4.304.31) in a thermal state at temperature $T=$ $\beta^{-1}$. The KMS condition relates the two Wightman functions,

$$
G_{+}^{\beta}(z-i \beta, \vec{x}, \vec{y})=G_{-}^{\beta}(z, \vec{x}, \vec{y}) .
$$

There is another relation between them, which comes from the vanishing of the commutator at spacelike separations: if $\vec{x} \neq \vec{y}$, it follows from the second equality in (4.31) that at sufficiently small $t=t_{x}-t_{y}, G_{+}(t, \vec{x}, \vec{y})=G_{-}(t, \vec{x}, \vec{y})$. Using these two relations, $G_{ \pm}^{\beta}(t, \vec{x}, \vec{y})$ can be extended to define a single function on the complex plane (for fixed $\vec{x}, \vec{y}), \mathcal{G}^{\beta}(z, \vec{x}, \vec{y})$, which satisfies the periodicity

$$
\mathcal{G}^{\beta}(z, \vec{x}, \vec{y})=\mathcal{G}^{\beta}(z+i n \beta, \vec{x}, \vec{y})
$$

for $n \in \mathbb{Z}$, and is holomorphic in the complex $z$ plane away from the lines $\Re(z)^{2}>$ $|\vec{x}-\vec{y}|^{2}, \Im(z)=n \beta$. A picture of this pole structure for a massive scalar field is given in figure [7. It is this characterisation of the thermal state in terms of the two-point function which is most useful and most commonly used in discussing quantum field theory in curved spacetime.

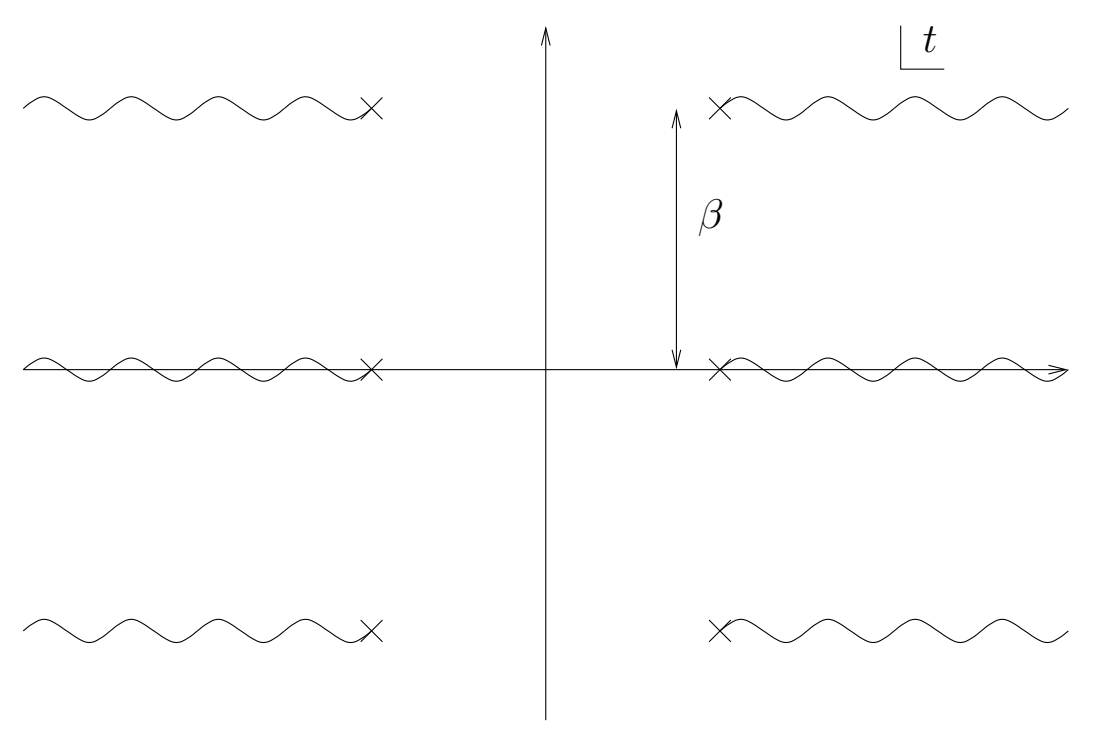

Figure 7: The pole structure of $\mathcal{G}^{\beta}(t, \vec{x}, \vec{y})$ for a massive scalar field in the complex $t$ plane for fixed values of $\vec{x} \neq \vec{y}$. The crosses represent poles and the wavy lines are branch cuts.

A thermal state for a free scalar field on a stationary spacetime $\mathcal{M}=\mathbb{R}_{t} \times \Sigma$ is defined by a two-point function $\mathcal{G}^{\beta}(z, \vec{x}, \vec{y})$ satisfying the periodicity condition (4.33). In particular, if the spacetime is static, so that the analytic continuation $t \rightarrow i \theta$ defines a Euclidean spacetime $\mathcal{M}_{E}=\mathbb{R}_{\theta} \times \Sigma$, Green's functions $G^{\beta}(\theta, \vec{x}, \vec{y})$ on the Euclidean spacetime periodic under $\theta \rightarrow \theta+\beta$ are related by analytic continuation to thermal states on the original Lorentzian spacetime. 


\section{Unruh \& Hawking radiation}

We now turn to the central task, demonstrating that event horizons have thermal properties for a quantum field in curved spacetime.

\subsection{Unruh radiation}

We consider first the thermal Unruh radiation associated to the Rindler horizon in flat spacetime. This is the simplest example of a bifurcate Killing horizon, and as discussed in section 2.2, it provides a good approximation to the geometry in the neighbourhood of a generator of an arbitrary Killing horizon. Hence, this case contains the essential physics. In flat spacetime, we have a preferred state for a quantum field, namely the Minkowski vacuum. We wish to show that this state will appear thermal with respect to the Rindler time. This result was originally derived by Unruh [62, following Hawking's derivation of Hawking radiation in [13, 14, and is therefore called the Unruh effect.

Recall that the Rindler coordinates $(x, t)$ were related to the usual Cartesian coordinates $(T, X)$ on flat space by (2.20),

$$
X=x \cosh \kappa t, \quad T=x \sinh \kappa t
$$

so we have

$$
d s^{2}=-d T^{2}+d X^{2}+d s_{\mathbb{R}^{(d-2)}}^{2}=-\kappa^{2} x^{2} d t^{2}+d x^{2}+d s_{\mathbb{R}^{(d-2)}}^{2} .
$$

Another useful coordinate system can be defined by setting $x=e^{\kappa \rho}$, so

$$
d s^{2}=\kappa^{2} e^{2 \kappa \rho}\left(-d t^{2}+d \rho^{2}\right)+d s_{\mathbb{R}^{(d-2)}}^{2}=-2 \kappa^{2} e^{2 \kappa(u-v)} d u d v+d s_{\mathbb{R}^{(d-2)}}^{2},
$$

where $u, v=t \pm \rho$. These null coordinates are related to the Cartesian ones $U, V=$ $T \pm X$ by $U=e^{\kappa u}, V=-e^{-\kappa v}$.

We consider the two-point function in the Minkowski vacuum,

$$
G_{M}\left(T_{X}-T_{Y}, X, Y\right)=\left\langle 0\left|\varphi\left(x^{\mu}\right) \varphi\left(y^{\mu}\right)\right| 0\right\rangle
$$

We could use the explicit form of this two-point function and apply the above coordinate transformations directly to show that, written in terms of the Rindler coordinates,

$$
G_{M}\left(T_{X}-T_{Y}, X, Y\right)=G_{R}^{2 \pi / \kappa}\left(t_{x}-t_{y}, x, y\right)
$$

that is, that the resulting function has period $2 \pi / \kappa$ in the complex $t_{x}-t_{y}$ plane. However, to avoid writing lengthy formulae and to demonstrate the simplicity and inevitability of the result, we will instead argue for this result more indirectly.

Consider the extension of $G_{M}\left(T_{X}-T_{Y}, X, Y\right)$ to a complex function in the complex $Z=T_{X}-T_{Y}$ plane. This function will be holomorphic for $\Im(Z) \neq 0$, as shown in figure 8. In particular, if we consider $Z=i\left(W_{X}-W_{Y}\right)$, this will define a regular Green's function

$$
G_{E}\left(W_{X}-W_{Y}, X, Y\right)=G_{M}(Z, X, Y)
$$




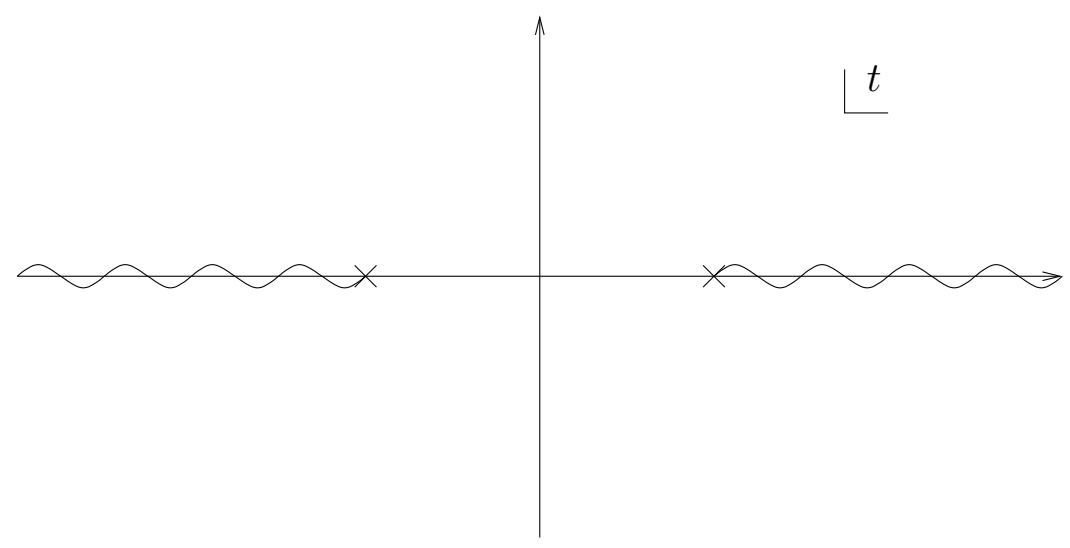

Figure 8: The pole structure of $G_{M}$.

satisfying

$$
\left(\square_{E}-m^{2}\right) G_{E}=\delta^{d}\left(x^{\mu}-y^{\mu}\right)
$$

on the Euclidean flat space defined by analytically continuing $T \rightarrow i W$,

$$
d s^{2}=d W^{2}+d X^{2}+d s_{\mathbb{R}^{(d-2)}}^{2} .
$$

This is just the inversion of familiar statement that the vacuum two-point function can be defined by Wick rotation from the Euclidean Green's function. Depending on how we choose to approach the axis, this defines the Feynman propagator, one of the Wightman functions, or some other propagator, but in any case, the Euclidean Green's function specified by (5.7) is uniquely defined.

Now we define new coordinates on the Euclidean space by $W=x \sin \kappa \tau, X=$ $x \cos \kappa \tau$, so

$$
d s^{2}=\kappa^{2} x^{2} d \tau^{2}+d x^{2}+d s_{\mathbb{R}^{(d-2)}}^{2} .
$$

Clearly $G_{E}\left(\tau_{x}-\tau_{y}, x, y\right)$ is a periodic function of $\tau_{x}-\tau_{y}$ with period $2 \pi / \kappa$. But this spacetime is also the result of analytically continuing the Rindler time coordinate $t \rightarrow i \tau$; that is, once we analytically continue to the Euclidean section, the coordinate transformation (2.20) relating Rindler and flat coordinates becomes the relation of Cartesian to polar coordinates. The analytic continuation of the boost isometry which defined the Rindler time translation is a rotation. Hence, if we now extend $G_{E}\left(\tau_{x}-\tau_{y}, x, y\right)$ to a holomorphic function of a complex coordinate $z=\tau_{x}-\tau_{y}$, we get

$$
\left.G_{E}(z, x, y)\right|_{z=-i\left(t_{x}-t_{y}\right)}=G_{R}^{2 \pi / \kappa}\left(t_{x}-t_{y}, x, y\right),
$$

and we have verified that the Minkowski vacuum is indeed a thermal state with respect to Rindler time with temperature $T=\kappa / 2 \pi$. We see that from this point of view, the thermal behaviour arises from the analytic continuation relation between Rindler coordinates in Minkowski space and polar coordinates in the Euclidean section.

We have discussed the case of a free scalar field, but the result is far more general: Bisognano and Wichmann [63, 64] proved a theorem which implies (as shown in [65]) that the Minkowski vacuum satisfies the KMS condition with respect to the boost time-translation Killing vector for an arbitrary interacting field theory on flat space. 
There is still an unanswered question, however: the Minkowski vacuum is a pure state, so how can it be transmuted into a thermal state by simply making a change of coordinates? This thermal behaviour actually comes from the fact that a Rindler observer, following an orbit of $\partial_{t}$, cannot see the whole spacetime. Thus, to describe the state as seen by such an observer, we should trace over the degrees of freedom in the unseen region of spacetime. This produces a mixed state describing the Rindler observer's measurements, with entropy coming from the entanglement between modes in the two sides of the spacetime.

To describe this entanglement, we need to relate the basis in the space of solutions which is appropriate for a Rindler observer to the usual Minkowski modes. The Minkowski modes are the usual plane-wave modes

$$
u_{k}=\psi_{k} e^{-i \omega_{k} T}
$$

where $\psi_{k}$ is a spatial wavefunction whose explicit form we do not need, and $\omega_{k}$ is a positive frequency. The Minkowski vacuum $|0\rangle$ is defined by $a_{k}|0\rangle=0$ for all $k$, where $a_{k}=\left(u_{k}, \varphi\right)$ is the annihilation operator associated with the mode $u_{k}$. The Rindler modes are defined by

$$
\begin{aligned}
& { }^{R} u_{k}=\left\{\begin{array}{l}
\tilde{\psi}_{k} e^{-i \omega t} \text { in } \mathrm{R}, \\
0 \quad \text { in } \mathrm{L},
\end{array}\right. \\
& { }^{L} u_{k}=\left\{\begin{array}{l}
0 \text { in } \mathrm{R}, \\
\tilde{\psi}_{k} e^{i \omega t} \text { in } \mathrm{L},
\end{array}\right.
\end{aligned}
$$

where $\mathrm{L}$ and $\mathrm{R}$ refer to the two wedges in figure 4 .

The Minkowski vacuum contains correlations between the $\mathrm{L}$ and $\mathrm{R}$ Rindler modes. Understanding the relation between the modes is simplified by using an observation due to Unruh [62, which emphasises the physics of the correlations. For simplicity, I will describe this in the case $m^{2}=0$; the extension to massive fields is left as an exercise.

For $m^{2}=0$ the positive frequency Rindler mode in the $\mathrm{R}$ wedge is simply ${ }^{4}$

$$
p=\left\{\begin{array}{l}
e^{-i \omega v} \quad \text { in } \mathrm{R}, \\
0 \quad \text { in L. }
\end{array}\right.
$$

The mode $p$ is not purely positive frequency with respect to the Minkowski time $T$. However, Unruh observed that we can construct a solution which is purely positive frequency with respect to $T$, and which agrees with $p$ in the $\mathrm{R}$ wedge. Write $p$ in Minkowski coordinates, as

$$
p=\left\{\begin{array}{l}
e^{i \omega \ln (-V) / \kappa} \text { for } V<0 \\
0 \quad \text { for } V>0
\end{array}\right.
$$

\footnotetext{
${ }^{4}$ The mode has the same form as a plane wave mode in Cartesian coordinates, as the $\mathrm{R}$ wedge is conformal to a flat space (as we can see from (5.3)). The frequency with respect to the Rindler time coordinate $t$ is $\omega$. However, unlike the usual plane wave modes, the physical energy of the mode redshifts with $x$. That is, the energy measured by a given Rindler observer will be $E_{\text {local }}=\omega /(\kappa x)$. Hence, if we considered an outgoing wavepacket, which will follow the null lines of constant $U$, it will redshift as it propagates to larger $x$.
} 
Then

$$
P=\left\{\begin{array}{l}
e^{i \omega \ln (-V) / \kappa} \quad \text { for } V<0 \\
e^{-\pi \omega / \kappa} e^{i \omega \ln (V) / \kappa} \quad \text { for } V>0
\end{array}\right.
$$

is the required mode solution; it clearly agrees with $p$ for $V<0$, and it is purely positive frequency, as it is analytic in the lower-half $V$ plane. We can also rewrite this as

$$
P=p+e^{-\pi \omega / \kappa} \tilde{p},
$$

where

$$
\tilde{p}=\left\{\begin{array}{l}
0 \quad \text { in } \mathrm{R}, \\
e^{-i \omega v} \quad \text { in } \mathrm{L},
\end{array}\right.
$$

is the result of 'flipping' the mode $p$ over the line $V=0$; it is a negative-frequency Rindler mode with support in the L wedge.

We now use the positive-frequency mode $P$ to show that there are correlations between $\mathrm{L}$ and $\mathrm{R}$ Rindler modes in the Minkowski vacuum. Since $P$ has positive frequency with respect to $T, a(P)=(P, \varphi)$ satisfies $a(P)|0\rangle=0$. As

$$
a(P)=a(p)+e^{-\pi \omega / \kappa} a(\tilde{p})=a(p)-e^{-\pi \omega / \kappa} a^{\dagger}\left(\tilde{p}^{*}\right),
$$

we can rewrite this as

$$
a(p)|0\rangle=e^{-\pi \omega / \kappa} a^{\dagger}\left(\tilde{p}^{*}\right)|0\rangle,
$$

demonstrating the existence of correlations. If we repeat this argument for a similar positive frequency mode defined starting from the $\mathrm{L}$ wedge,

$$
P^{\prime}= \begin{cases}e^{-\pi \omega / \kappa} e^{-i \omega \ln (-V) / \kappa} \quad \text { for } V<0 \\ e^{-i \omega \ln (V) / \kappa} & \text { for } V>0,\end{cases}
$$

we can conclude that

$$
a\left(\tilde{p}^{*}\right)|0\rangle=e^{-\pi \omega / \kappa} a^{\dagger}(p)|0\rangle .
$$

These two relations (5.2015.22) can be solved by writing

$$
|0\rangle=\exp \left[e^{-\pi \omega / \kappa} a^{\dagger}\left(\tilde{p}^{*}\right) a^{\dagger}(p)\right]\left|0_{p \tilde{p}^{*}}\right\rangle,
$$

where $\left|0_{p \tilde{p}^{*}}\right\rangle$ is a vacuum state for the modes $p, \tilde{p}^{*}$; that is, a state satisfying

$$
a\left(\tilde{p}^{*}\right)\left|0_{p \tilde{p}^{*}}\right\rangle=a(p)\left|0_{p \tilde{p}^{*}}\right\rangle=0 .
$$

If we expand out the exponential,

$$
|0\rangle=\sum_{m} e^{-\pi \omega_{m} / \kappa}|m\rangle_{L} \times|m\rangle_{R}
$$

where $|m\rangle_{R, L}$ are states with $m$ particles in the mode $p\left(\tilde{p}^{*}\right)$ in the $\mathrm{R}(\mathrm{L})$ wedge, and $\omega_{m}$ is the corresponding energy. Thus, from the point of view of Rindler observers, the Minkowski vacuum $|0\rangle$ contains correlated pairs of particles in the modes $p, \tilde{p}^{*}$ : that is, it is a squeezed state with respect to the Rindler basis. 
This analysis applies to an arbitrary Rindler mode. Formally, we can solve all the relations by writing

$$
|0\rangle=\prod_{i} \exp \left[e^{-\pi \omega_{i} / \kappa} a^{\dagger}\left(\tilde{p}_{i}^{*}\right) a^{\dagger}\left(p_{i}\right)\right]|0\rangle_{L} \times|0\rangle_{R}
$$

for some basis $p_{i}$ of Rindler modes, where $|0\rangle_{L, R}$ are vacuum states wrt to the Rindler modes. However, it should be borne in mind that this is just a formal expression; the Minkowski vacuum is not in the same Hilbert space as the Rindler vacuum.

If we consider $\left\langle 0\left|\mathcal{O}_{R}\right| 0\right\rangle$ for some operator $\mathcal{O}_{R}$ which acts only on the $\mathrm{R}$ wedge, we trace over the degrees of freedom in the $\mathrm{L}$ wedge, and the correlations between particles in the $\mathrm{L}$ and $\mathrm{R}$ wedges will give rise to thermal behaviour for the expectation value: formally,

$$
\left\langle 0\left|\mathcal{O}_{R}\right| 0\right\rangle=\sum_{m} e^{-2 \pi \omega_{m} / \kappa}\left\langle m\left|\mathcal{O}_{R}\right| m\right\rangle
$$

where the sum runs over a complete basis $|m\rangle$ in the Fock space built on $|0\rangle_{R}$.

This analysis provides a second, more intuitive point of view on the thermal nature of the Minkowski vacuum from the Rindler observers' perspective. Its generalisation will be important in studying Hawking radiation for a general black hole. The first calculation, however, avoids reference to any particular mode decomposition, and highlights an intriguing connection with Euclidean spacetime.

\subsection{Hawking radiation}

We turn now to the thermodynamic properties of black holes. We will first consider the eternal black hole, and show that there is a generalisation of the above discussion of Rindler which applies to the Killing horizon in a stationary black hole spacetime. However, we should note at the outset that this is not really relevant to black holes formed by gravitational collapse in asymptotically flat space; we briefly discuss the argument for Hawking radiation in this truly dynamical situation at the end of the section.

We consider for simplicity the Schwarzschild black hole (2.1). As in flat space, we have a time-translation $\partial_{t}$ which is timelike in the two wedge regions on the left and right in figure 1. If we complexify this time coordinate by $t \rightarrow i \tau$, we obtain the Euclidean metric

$$
d s^{2}=\left(1-\frac{2 M}{r}\right) d \tau^{2}+\left(1-\frac{2 M}{r}\right)^{-1} d r^{2}+r^{2}\left(d \theta^{2}+\sin ^{2} \theta d \phi^{2}\right) .
$$

In this metric, $r=2 M$ is an origin in the $r, \tau$ plane. The spacetime is smooth there if $\tau$ is an angular coordinate with period $\beta=2 \pi / \kappa$ where $\kappa=1 / 4 M$ is the black hole's surface gravity. This gives us the 'semi-infinite cigar' geometry for the Euclidean black hole depicted in figure 9.

If we define a quantum state by taking the Euclidean Green's function $G(x, y)$ on this spacetime and analytically continuing it to obtain a two-point function on the Lorentzian black hole spacetime, the resulting two-point function $G^{\beta}\left(t_{x}-t_{y}, x, y\right)$ will 


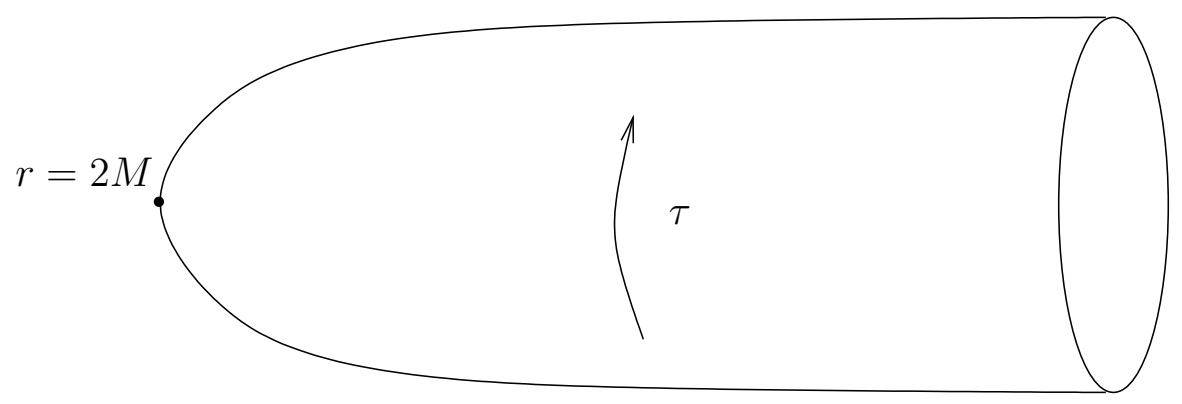

Figure 9: The Euclidean black hole geometry, suppressing the two-sphere directions $\theta, \phi$.

satisfy the KMS condition by the same argument used for the Unruh radiation above. That is, the state defined by analytic continuation from the Euclidean black hole, which is referred to as the Hartle-Hawking state, is a thermal state at temperature $T=\kappa / 2 \pi$. The temperature is fixed by the periodicity of $\tau$ required to make the Euclidean space smooth at the horizon [66].

In Rindler space, the analogous state was selected as a preferred state because it was the Minkowski vacuum. In the Schwarzschild solution, however, there is no global notion of time-translation - that is, there is no Killing vector which is timelike everywhere in figure 1 so there is no natural notion of vacuum state. How then do we choose the Hartle-Hawking state from the Lorentzian point of view?

The answer is again to demand regularity at the horizon. There is a theorem due to Kay and Wald [58] which states that for the free scalar field on the Schwarzschild geometry, the thermal state at temperature $T=\kappa / 2 \pi$ is the unique Hadamard state invariant under $\partial_{t}$. Thus, the only thing that can be in equilibrium with the black hole is thermal radiation at temperature $T$. More generally, [58] show that if there exists an invariant Hadamard state on a stationary spacetime with a bifurcate Killing horizon, it is a KMS state with temperature $T=\kappa / 2 \pi$; in particular, the discussion carries through in exactly the same way for Schwarzschild-AdS. Note however that in generalising the black hole solutions we consider, it can easily happen that no invariant Hadamard state exists. For example, none does for Kerr: this is connected to the superradiance property for Kerr.

Another important point to consider is whether the Hartle-Hawking state is physically relevant. There are two issues here: first, this state involves a particular boundary condition at infinity. It contains an equal flux of incoming and outgoing thermal radiation (as the state is symmetric under $t \rightarrow-t$ ). As we will argue later, this is a good approximation to the late-time behaviour of a black hole in asymptotically AdS space, but not in asymptotically flat space. Second, we should ask if it describes a stable equilibrium. As noted previously, a KMS state is locally dynamically stable, but the question is now whether the black hole is stable to small fluctuations. For the Schwarzschild black hole described above, it is not; since $T=1 / 8 \pi M$, the specific heat $C_{V}=\partial M / \partial T<0$. Thus, if the mass fluctuates downwards, the temperature rises, and the black hole will radiate more than it absorbs from the thermal bath, further lowering its mass. So this equilibrium state for Schwarzschild is unphysical; 
real black holes will never reach this equilibrium (even if they are illuminated with a thermal flux of incoming radiation).

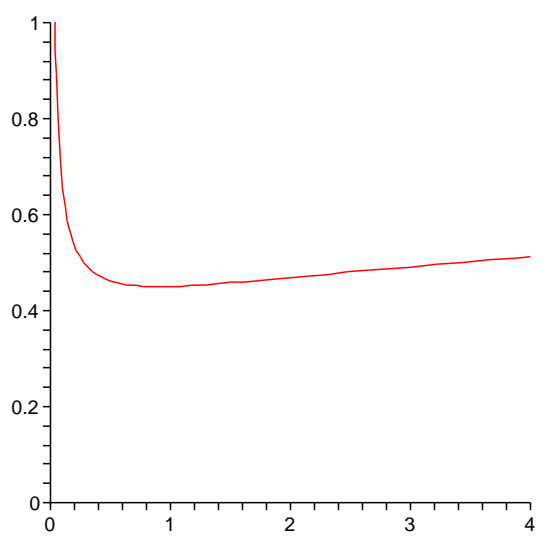

Figure 10: The temperature for Schwarzschild-AdS black holes as a function of mass, for the case $d=5$. ( $T \ell$ is plotted against $G M / \ell^{2}$.)

For Schwarzschild-AdS black holes, on the other hand, the temperature is

$$
T=\frac{(d-1) r_{+}^{2}+(d-3) \ell^{2}}{4 \pi r_{+} \ell^{2}} .
$$

For $r_{+} \ll \ell$, the behaviour is similar to the Schwarzschild case, but for $r_{+}^{2} / \ell^{2}=$ $(d-3) /(d-1)$, we reach a minimum temperature, $T_{\min }=\sqrt{(d-1)(d-3)} / 2 \pi \ell$, and for $r_{+} \gg \ell$

$$
T=\frac{(d-1)}{4 \pi \ell^{2}} r_{+} \propto M^{\frac{1}{d-1}}
$$

so the specific heat becomes positive. The temperature is plotted as a function of mass in figure 10. Thus for large black hole mass this becomes a stable equilibrium, and really represents the endstate of black hole plus radiation.

So far, we have discussed quantum fields on the stationary black hole spacetimes, and analysed the curved space version of the Unruh effect. However, in the real world, we are interested in the behaviour of a black hole which forms by the gravitational collapse of a massive body, as sketched in figure 11. We will now briefly describe the argument that such a black hole will emit thermal radiation at late times - the true Hawking effect.

Once the collapsing matter has crossed the event horizon, the geometry near the horizon will quickly settle down, approaching an approximately stationary geometry, which is approximately vacuum on and outside the event horizon. ${ }^{5}$ This implies that

\footnotetext{
${ }^{5}$ Note that it seems that this assumption will fail for black holes in asymptotically flat space, once we take into account the back-reaction of the emitted Hawking radiation, which causes the black hole to lose mass, balancing the energy lost to the outgoing radiation. However, this will still be a good approximation over timescales shorter than the time $t_{b r} \sim\left(M / M_{p l}\right)^{3}$ over which Hawking radiation carries away an appreciable fraction of the mass. For astrophysical black holes, this is a very long timescale.
} 


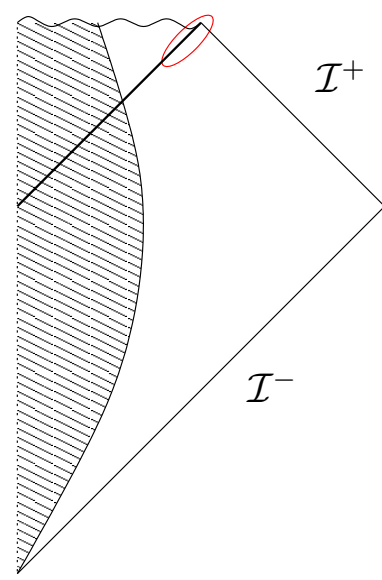

Figure 11: The Penrose diagram of the formation of a black hole by gravitational collapse in asymptotically flat space. The argument for the Hawking effect involves focusing on the circled region.

the geometry of any small neighbourhood of the future event horizon is approximately flat space, and the stationary observers outside the black hole are approximately the Rindler observers in flat space by the argument of section 2.2

Now comes the crucial assumption: we assume that the quantum state of the scalar field $\varphi$ on the black hole geometry behaves locally in the same way as the Minkowski vacuum. That is, freely-falling observers near the horizon should not see any unusual behaviour in high-energy processes. ${ }^{6}$ We are essentially again assuming the state is regular (Hadamard) at the event horizon. We can then conclude that the Rindler modes on either side of the horizon are correlated as in (5.2015.22), and the approximately Rindler observers outside the horizon will see outgoing thermal radiation at the temperature (with respect to $\partial_{t}$ )

$$
T_{H}=\frac{\hbar \kappa}{2 \pi} .
$$

However, unlike in our previous discussion, this black hole will not be in thermal equilibrium with this radiation. This is because the natural boundary condition on past null infinity $\mathcal{I}^{-}$is that the state approach the Minkowski vacuum. Thus, there is outgoing thermal radiation, but no ingoing radiation. The collapse geometry breaks the $t \rightarrow-t$ symmetry, even when we concentrate on the behaviour long after collapse has taken place. The stationary state on the eternal black hole which satisfies these boundary conditions is called the Unruh state (it is not Hadamard on the past horizon). At late times, a black hole formed from gravitational collapse is well-approximated by the eternal black hole with the scalar field in the Unruh state, and not the Hartle-Hawking state. If we take into account back-reaction, the black

\footnotetext{
${ }^{6}$ An interesting question in this dynamical context is how this free-fall vacuum arises. If we trace the outgoing Hawking quanta backwards, then due to the redshift noted previously for the Rindler modes, they will originate in modes of trans-Planckian frequencies before the collapse. See [28] for a nice review of this trans-Planckian problem.
} 
hole will slowly reduce in mass, losing its energy to the outgoing Hawking radiation, so it never reaches an equilibrium configuration.

The situation is different for an asymptotically AdS black hole. The asymptotically AdS boundary conditions act as a reflecting box, and the outgoing Hawking quanta are reflected back towards the black hole. As a result, at late times the black hole can come to equilibrium with its Hawking radiation, and for a sufficiently large black hole, the late-time behaviour is well-approximated by the eternal black hole with the scalar field in the Hartle-Hawking state.

In this section we have seen that a quantum field in equilibrium with a black hole must be in a thermal state at temperature $T=\frac{\hbar \kappa}{2 \pi}$, where $\kappa$ is the black hole's surface gravity. This is the key result which converts the analogy between black hole mechanics and thermodynamics in section 3 into an equivalence. We can combine this temperature with the first law that we demonstrated classically in section 3.2 to argue that the black hole has an entropy $S=\frac{A}{4 G \hbar}$.

\section{Euclidean entropy calculation}

The argument given above provides solid evidence for black hole thermodynamics. It uses a rigorously defined theory, quantum field theory on a fixed classical spacetime background. Since the geometry at the event horizon can be taken to have arbitrarily small curvature in Planck units, this should be a good approximation to the true physical description of the situation. In the AdS case, where there is a stable equilibrium between the black hole and its radiation, corrections to this description should be small on and outside the horizon for all times.

However, having established that the black holes have entropy $S=A / 4 G \hbar$, we would like to understand why entropy and area are related in this way. In this section, I will review the Euclidean path integral approach, which provides a very general connection between entropy and area, and discuss the description of black holes in string theory via the AdS/CFT correspondence using this Euclidean point of view. The discussion in this section is somewhat briefer and more sketchy than previously. In particular, have no intention of providing a review of AdS/CFT; the reader should consult the lectures by Leonardo Rastelli in this volume, or one of the other reviews (e.g., [18]).

A very general connection between entropy and geometry has been established in the Euclidean path integral approach to quantum gravity (see [67, 68, for reviews). In this approach, the canonical partition function for the gravitational field is defined by a sum over all smooth Riemannian geometries which are periodic with period $\beta=T^{-1}$ in imaginary time,

$$
Z(\beta)=\int d[g] e^{-I[g]}
$$

where $I[g]$ is the classical action of the geometry. In the asymptotically flat context, what this means is that the integration in (6.1) includes all asymptotically flat geometries with an isometry along a compact direction whose proper size at infinity 
is $\beta$. In the asymptotically $\mathrm{AdS}$ context, if we identify the time coordinate of the Euclidean black hole solution (2.9) periodically, the proper size of this $S^{1}$ will tend to infinity at large distances. In this case the relevant quantity to fix is the asymptotic value of the dimensionless ratio of the size of this $S^{1}$ to the size of the $S^{d-2}$. In both cases, it is important to note that we only impose an asymptotic boundary condition on the metric; the metric in the bulk of the spacetime is allowed to fluctuate.

There are problems with the definition of this Euclidean path integral: these include the non-renormalisable ultraviolet divergences of gravity, the indefiniteness of the gravitational action, which is not even bounded from below, and the fact that the on-shell action typically diverges for non-compact solutions. I will adopt the view that the path-integral expression is merely a semi-classical tool. That is, one should not view the sum over geometries as a fundamental definition of the theory; instead, we are interested in seeing what insight we can gain from considering the saddle-point approximation to this integral, where we approximate

$$
\ln Z(\beta) \approx-I_{s},
$$

where $I_{s}$ is the classical action of a Euclidean solution which satisfies the boundary conditions. There may be more than one such solution; we consider the dominant contribution, which comes from the solution of least action. The expectation is that this approximation should give useful results if the classical solution is weakly curved, whatever the fundamental quantum theory may be. This expectation is magnificently borne out in the AdS/CFT context, as we will see a little later. Since $Z(\beta)$ is the canonical partition function, $Z(\beta)=e^{-\beta F}=e^{-\beta\langle E\rangle+S}$, we can evaluate the energy and entropy by the standard formulae

$$
\begin{gathered}
\langle E\rangle=-\frac{\partial}{\partial \beta} \ln Z \approx \frac{\partial}{\partial \beta} I_{s}, \\
S=\beta\langle E\rangle+\ln Z=-\left(\beta \frac{\partial}{\partial \beta}-1\right) \ln Z \approx\left(\beta \frac{\partial}{\partial \beta}-1\right) I_{s} .
\end{gathered}
$$

There is an important topological difference between the Euclidean solutions which do and do not involve black holes. In for example the Euclidean flat space,

$$
d s^{2}=d \tau^{2}+d r^{2}+r^{2} d \Omega_{d-2},
$$

the Killing vector $\partial_{\tau}$ is non-vanishing throughout the spacetime. The radial coordinate $r$ ranges over $r \geq 0$, and the $S^{d-2}$ shrinks to zero size at $r=0$. We can identify $\tau$ periodically with any period we choose. On the other hand, for the Euclidean Schwarzschild solution (5.28), the Killing vector $\partial_{\tau}$ vanishes at $r=r_{+}=2 M$, which is a fixed point of the isometry. We must take $r \geq r_{+}$, and identify $\tau$ periodically with period $2 \pi / \kappa$ to obtain a smooth geometry. The $S^{1}$ shrinks to zero size at $r=r_{+}$. This fixed point of $\partial_{\tau}$ is the Euclidean continuation of the bifurcate Killing horizon in the Lorentzian black hole solution.

For cases with no black hole, where the circle direction does not shrink to zero, we can exploit the fact that global time is a Killing symmetry to write the action as

$$
I=\int d^{d} x \mathcal{L}=\int d \tau \int d^{d-1} x \mathcal{L}=\beta H,
$$


where $H$ is the Hamiltonian. Hence, when such a geometry provides the dominant saddle point, $\ln Z \approx I$ is linear in $\beta$, and

$$
S \approx\left(\beta \frac{\partial}{\partial \beta}-1\right) I=0
$$

That is, there is no classical contribution to the entropy for this solution, as we would expect.

For solutions with a black hole, on the other hand, such a foliation by surfaces of constant time will necessarily break down in the interior, where the $S^{1}$ degenerates. Thus, the action will not be linear in $\beta$. We can split the integration over the spacetime up in the way shown in figure 12, into an integral over a small disc around the horizon at $r=r_{+}$, and the remaining integration. The remaining integration will then be linear in $\beta$, as this region can be foliated with surfaces of constant $t$.

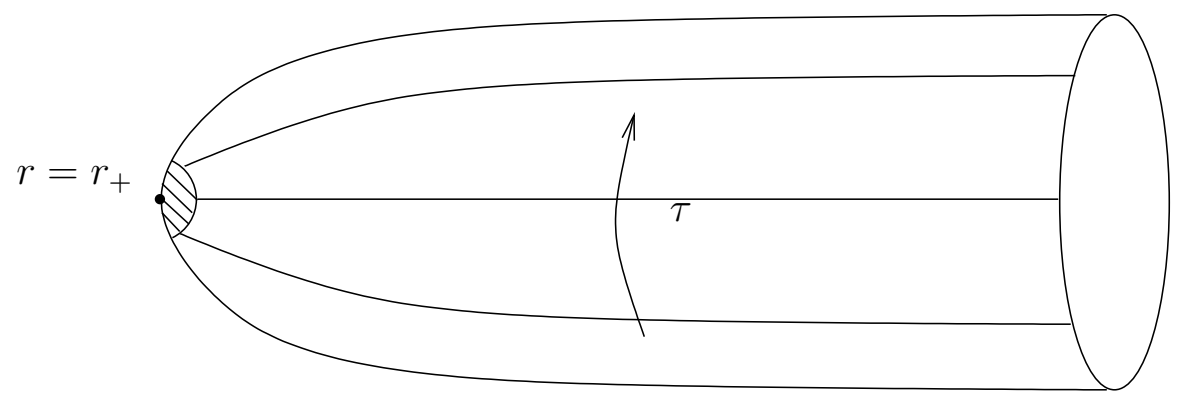

Figure 12: Decomposition of the calculation of the bulk action into a small region near the horizon and the remainder.

One might think that the integration over the small disc would vanish in the limit as we take the size of the disc to zero, since this is a smooth region of spacetime. However, this is not the case: to be able to write the integration over the bulk of the spacetime in Hamiltonian form, we have to be careful about how we break up the integration, which means we have to keep a boundary term in the action (see [69, 70]). The (leading-order) gravitational part of the action for the disc is

$$
I_{\text {grav }}=\frac{1}{16 \pi G} \int_{M} d^{d} x \sqrt{-g} R+\frac{1}{8 \pi G} \int_{\partial M} d^{d-1} y \sqrt{-h} K .
$$

The first term is the usual Einstein-Hilbert term; the second term is the integral of the trace of the extrinsic curvature over the boundary, $K=h^{\mu \nu} \nabla_{\mu} n_{\nu}$, where $n^{\mu}$ is the normal to and $h_{\mu \nu}$ the induced metric on the boundary. The surface term can also be rewritten as

$$
\int_{\partial M} d^{d-1} y \sqrt{-h} K=-\frac{\partial}{\partial n} \int_{\partial M} d^{d-1} y \sqrt{-h} .
$$

This surface term is necessary to ensure that the variation of the action vanishes under arbitrary variations of the metric which vanish on $\partial M$ [71.

For a small disc near the horizon, the metric is approximately

$$
d s^{2} \approx \rho^{2} \kappa^{2} d \tau^{2}+d \rho^{2}+r_{+}^{2} d \Omega
$$




$$
\int_{r=r_{+}+\epsilon} d^{d-1} y \sqrt{-h}=2 \pi \epsilon A,
$$

where $A$ is the area of the horizon, that is, the volume of an $S^{d-2}$ of radius $r_{+}$, and

$$
\frac{\partial}{\partial n} \int_{\partial M} d^{d-1} y \sqrt{-h}=2 \pi A \text {. }
$$

Hence, in the limit $\epsilon \rightarrow 0$, the small disc around $r=r_{+}$makes a contribution

$$
I_{\text {disc }}=-\frac{1}{4 G} A
$$

which gives

$$
S=\left(\beta \frac{\partial}{\partial \beta}-1\right) I_{s}=\left(\beta \frac{\partial}{\partial \beta}-1\right) I_{d i s c}=\frac{1}{4 G} A .
$$

This calculation provides a direct link between geometry and entropy. It shows that the non-zero entropy associated to a black hole is a consequence of the non-trivial topology of the Euclidean solution. As in the calculation of the temperature in quantum field theory, regularity of the geometry at the horizon plays a key role in the derivation. Note that the explicit form of the geometry was not used in this derivation, just the fact that the geometry is smooth there. Thus, this derivation explains the universality of the relation between entropy and area.

Note also that the explicit form of the action is used here, so the result depends on the gravitational dynamics (unlike the calculation of the temperature, which is more kinematical). This Euclidean calculation has been shown to be equivalent to the Noether charge construction of the entropy appearing in the first law [72]. Hence for a more general action, we would produce corrections to the above area formula, as in the discussion from the first law point of view in section 3.2

This saddle-point calculation of the black hole entropy does not offer any insight into the nature of the microstates the black hole entropy is counting. However, in black hole pair creation, a similar instanton approximation provides evidence that the black hole entropy is really counting microstates (see [73] for a review). There are processes where a pair of black holes can be created through quantum tunnelling: for example, a pair of charged black holes can be created in a sufficiently intense electric or magnetic field. The pair creation rate can be estimated by instanton methods, using a similar Euclidean saddle-point. The rate is $\Gamma \sim e^{-I_{s}}$, where $I_{s}$ is the action of the Euclidean instanton. In this context, the additional contribution to the action associated with the event horizon corresponds to an enhancement of the pair creation rate by a factor of $e^{A / 4}$ relative to the pair creation rate of elementary objects of the same mass, just as we would expect if we were summing over the rate for black holes to be created with in each of $e^{A / 4}$ internal states [70].

To explicitly identify these microstates, we need a concrete microscopic theory of quantum gravity. I will now briefly describe the calculation of the entropy in the AdS/CFT correspondence. The fundamental relation between field theory and spacetime in any of the AdS/CFT correspondences is that

$$
\left\langle e^{\int \phi_{0} \mathcal{O}}\right\rangle=Z_{S}\left(\phi_{0}\right) \text {. }
$$


The LHS is the generating function of correlation functions in the field theory, which depends on sources $\phi_{0}$ coupled to the operators $\mathcal{O}$ of the theory. This is identified on the RHS with a string theory partition function in an asymptotically anti-de Sitter spacetime, with boundary conditions determined by $\phi_{0}$. The two most studied examples of this correspondence are: the string theory on $A d S_{5} \times S^{5}$, with $N$ units of RR five-form flux, is identified with $\mathcal{N}=4, S U(N)$ Yang-Mills theory in $d=4$, with coupling $g_{Y M}^{2} \sim g_{s}$. Since the curvature scale in $A d S_{5} \times S^{5}$ in string units goes like $g_{s} N$, the region where a semiclassical bulk calculation is a good approximation is $g_{Y M} \ll 1, \lambda=g_{Y M}^{2} N \gg 1$. The string theory on $A d S_{3} \times S^{3} \times X$ for $X=T^{4}$ or $K 3$, with $N_{1}$ units of electric RR 3-form flux and $N_{5}$ units of magnetic RR 3form flux, is identified a two-dimensional CFT with central charge $c=6 N_{1} N_{5}$, which is some deformation of the supersymmetric sigma-model on $X^{N_{1} N_{5}} / S_{N_{1} N_{5}}$. (These correspondences were proposed in [74] and elucidated in 75, 76]. See e.g. [18] for further details and references.)

We regard the CFT as providing the fundamental definition of the theory, while on the RHS, we are interested in the semi-classical approximation, or in perturbative calculation around some background. Thus, in particular, the canonical partition function in the CFT, the generating function for thermal correlation functions, is equal to the string theory version of the Euclidean quantum gravity partition function (6.1). ${ }^{7}$ Thus, the above saddle-point calculation provides a prediction from gravity for the behaviour of the entropy in the CFT at strong coupling.

In the CFT, we know precisely what this entropy is counting; it is a count of the states of a thermal gas of gluons in the field theory. In the $\mathrm{AdS}_{5}$ case, the gravitational calculation gives a free energy $F=-\frac{\pi^{2}}{8} N^{2} V T^{4}$, agreeing up to a numerical factor with the result obtained at weak coupling by counting the thermal excitations of the fundamental gauge theory fields, $F=-\frac{\pi^{2}}{6} N^{2} V T^{4}$ [77, 18]. In the $\mathrm{AdS}_{3}$ case, the spacetime calculation gives $S=2 \pi r_{+} / 4 G_{(3)}=2 \pi \sqrt{c E / 3}$, where $E=N_{L}+$ $N_{R}=2 N$ is the CFT energy (for a non-rotating black hole, $N_{L}=N_{R}=N$ ). This result can be precisely reproduced on the CFT side by using the Cardy formula $S=2 \pi\left(\sqrt{c N_{L} / 6}+\sqrt{c N_{R} / 6}\right)$ to calculate the entropy [78]. Thus, in this case, we obtain a precise agreement, including the numerical factor. The central charge of the CFT can be read off from the asymptotic isometries of the spacetime [79], so the agreement for $\mathrm{AdS}_{3}$ is largely independent of the detailed correspondence supplied by AdS/CFT.

Note that these results make no direct reference to the black hole horizon; the CFT description counts all the states with a given temperature in asymptotically AdS space. The result is related to the black hole entropy simply because for high enough temperature the black hole dominates this ensemble. This has the advantage that it avoids making any assumption about the spacetime near the horizon - we do not need to impose any boundary condition at the horizon. The drawback is that

\footnotetext{
${ }^{7}$ To generate thermal correlation functions in the CFT, we consider the Euclidean theory on $S^{1} \times S^{d-2}$, as in the previous discussion of the free scalar. The CFT is strongly interacting, but the argument that analytic continuation from this Euclidean space defines thermal correlators still applies.
} 
the spacetime interpretation of these microstates, and their relation to the geometry near the horizon, is obscure.

An interesting aspect of the gravitational calculation is that it predicts a phase transition as a function of temperature. The saddle-point which dominates the partition function in the approximation (6.2) can change as we vary the temperature. Recall the temperature for a Schwarzschild-AdS black hole is given by (5.29), so for $T<T_{\min }=\sqrt{(d-1)(d-3)} / 2 \pi \ell$, the only saddle point is thermal AdS. For $T>T_{\min }=\sqrt{(d-1)(d-3)} / 2 \pi \ell$, there are three saddle-points: thermal AdS, a small black hole with $r_{+}<\sqrt{\frac{(d-3)}{(d-1}} \ell$, and a large black hole with $r_{+}>\sqrt{\frac{(d-3)}{(d-1}} \ell$. If we define the action by background subtraction, so that $I=0$ for the Euclidean AdS solution by definition, we find

$$
I_{B H} \propto\left(\ell^{2} r_{+}^{d-2}-r_{+}^{d}\right)
$$

thus, the action of the black hole changes sign when $r_{+}=\ell$. When $T=(d-2) / 2 \pi \ell$, so the large black hole has $r_{+}=\ell$, there is a phase transition, the Hawking-Page transition [80, where the dominant contribution to the partition function changes from thermal AdS to the large black hole. The small black holes, which have negative specific heat, never make the dominant contribution to the partition function.

In the $\mathrm{AdS}_{5}$ case, this Hawking-Page transition corresponds to an expected phase transition in the dual four-dimensional field theory, the confinement-deconfinement transition, as discussed in 81. Thus, the qualitative thermodynamic behaviour agrees with the CFT expectations. The change in topology of the saddle plays an important role in the interpretation of the phase transition. Recently further work has been done studying the phase structure of the four-dimensional field theory at weak coupling, comparing it to this behaviour at strong coupling [82].

In the $\mathrm{AdS}_{3}$ case, we can calculate the elliptic genus of the CFT, which is a particular supersymmetry-protected partition function, exactly. In [83, it was shown that the result can be re-organized in a way which corresponds naturally to the contributions of different saddle-points in the bulk. Thus, in this case, the correspondence between the CFT and the sum over geometries in the bulk can be verified in detail.

There is thus a well-developed relation between the Euclidean black hole solutions and the thermal ensemble in the field theory. We can also describe the Lorentzian eternal black hole solution in AdS/CFT. The essential insight is to take the full Penrose diagram in figure 4 seriously. The natural dual description is then in terms of two copies of the CFT, one living on each of the two asymptotic boundaries. The black hole corresponds to an entangled state $|\Psi\rangle$ in the product Hilbert space which correlates the CFT modes living on the two boundaries [84, 85, 86,

$$
a_{1}|\Psi\rangle=e^{-\pi \omega / \kappa} a_{2}^{\dagger}|\Psi\rangle
$$

and vice-versa. That is, the whole black hole geometry is described by having an entanglement between the two CFTs of the same form as the entanglement between modes of a bulk field on the two sides of a Killing horizon (cf (5.20) ), even though the two boundaries cannot communicate through the bulk. This description can be 
derived by an analytic continuation from the Euclidean correspondence [86]. The entangled state $|\Psi\rangle$ is a standard representation of a thermal ensemble: tracing over one copy of the CFT gives a thermal density matrix in the other copy, so any observable which makes reference to only one boundary will have thermal expectation values. This proposed Lorentzian correspondence has been further developed by a number of authors [87, 88, 89, 90, 91, 92, 93, probing in particular the description of the region of spacetime 'behind the horizons'; that is, in the future and past wedges of figure 4.

With these recent insights from the AdS/CFT correspondence, we now have a fairly good picture of the relation between stationary black holes and thermal equilibrium. There are still interesting unresolved issues, such as a general discussion of the first law of thermodynamics for the black rings with 'dipole charges', but the most important open questions concern dynamical situations, such as the formation and evaporation of a small black hole in AdS. In AdS/CFT, relatively little progress has been made on these issues: we do not even have a good understanding of the CFT description of quasi-static changes in the geometry, let alone a dual description of the formation of a black hole from gravitational collapse. Addressing such dynamical questions is the key to addressing many important questions about black holes, notably the information loss problem [94. (see 95] for a review). Further progress on these issues will require a major step forward in our understanding of the AdS/CFT correspondence, building a dictionary relating bulk and boundary for dynamical spacetimes and moving away from the essentially Euclidean picture which has dominated our understanding to date.

\section{Acknowledgements}

I am grateful to the organisers of the meeting for the invitation to give these lectures, and I thank the students and other participants in the meeting for many sharp questions and interesting discussions. I thank Don Marolf and Veronika Hubeny for carefully reading the draft version of these notes. This work is supported by the EPSRC.

\section{References}

[1] T. Jacobson, "Introductory lectures on black hole thermodynamics." Given at Utrecht U. in 1996; available at

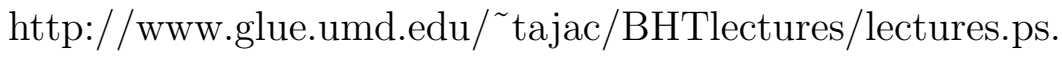

[2] R. M. Wald, "Black holes and thermodynamics," gr-qc/9702022.

[3] R. M. Wald, "The thermodynamics of black holes," Living Rev. Rel. 4 (2001) $6, \mathrm{gr}-\mathrm{qc} / 9912119$.

[4] J. H. Traschen, "An introduction to black hole evaporation," gr-qc/0010055.

[5] T. Padmanabhan, "Gravity and the thermodynamics of horizons," Phys. Rept. 406 (2005) 49-125, gr-qc/0311036. 
[6] D. N. Page, "Hawking radiation and black hole thermodynamics," hep-th/0409024.

[7] R. Brout, S. Massar, R. Parentani, and P. Spindel, "A primer for black hole quantum physics," Phys. Rept. 260 (1995) 329-454.

[8] S. A. Fulling and S. N. M. Ruijsenaars, "Temperature, periodicity and horizons," Phys. Repts. 152 (1987) 135.

[9] R. M. Wald, Quantum field theory in curved space-time and black hole thermodynamics. Chicago Univ. Press, Chicago, USA, 1994.

[10] S. M. Carroll, Spacetime and Geometry: An introduction to General Relativity. Addison Wesley, San Francisco, 2004.

[11] A. W. Peet, "TASI lectures on black holes in string theory," hep-th/0008241.

[12] J. R. David, G. Mandal, and S. R. Wadia, "Microscopic formulation of black holes in string theory," Phys. Rept. 369 (2002) 549-686, hep-th/0203048.

[13] S. W. Hawking, "Black hole explosions," Nature 248 (1974) 30-31.

[14] S. W. Hawking, "Particle creation by black holes," Commun. Math. Phys. 43 (1975) 199-220.

[15] G. 't Hooft, "Dimensional reduction in quantum gravity," gr-qc/9310026.

[16] L. Susskind, "The world as a hologram," J. Math. Phys. 36 (1995) 6377-6396, hep-th/9409089

[17] R. Bousso, "The holographic principle," Rev. Mod. Phys. 74 (2002) 825-874, hep-th/0203101.

[18] O. Aharony, S. S. Gubser, J. M. Maldacena, H. Ooguri, and Y. Oz, "Large N field theories, string theory and gravity," Phys. Rept. 323 (2000) 183-386, hep-th/9905111.

[19] R. H. Brandenberger, "Theory of cosmological perturbations and applications to superstring cosmology," hep-th/0501033.

[20] N. D. Birrell and P. C. W. Davies, Quantum fields in curved space. Cambridge Univ. Press, Cambridge, UK, 1982.

[21] R. Haag, Local quantum physics. Springer-Verlag, Berlin, 1992.

[22] S. A. Fulling, Aspects of quantum field theory in curved space-time, vol. 17 of London Mathematical Society student texts. Cambridge Univ. Press, Cambridge, UK, 1989.

[23] B. S. DeWitt, The global approach to quantum field theory., vol. 114 of Int. Ser. Monogr. Phys. Oxford Univ. Press, 2003. 2 vols. 
[24] B. S. Dewitt, "Quantum field theory in curved space-time," Phys. Rept. 19 (1975) 295-357.

[25] G. W. Gibbons, "Quantum field theory in curved space-time," in General Relativity: An Einstein centenary survey, S. W. Hawking and W. Israel, eds. Cambridge Univ. Press, Cambridge, UK, 1979.

[26] B. S. DeWitt, "Quantum gravity: The new synthesis," in General Relativity: An Einstein centenary survey, S. W. Hawking and W. Israel, eds. Cambridge Univ. Press, Cambridge, UK, 1979.

[27] L. H. Ford, "Quantum field theory in curved spacetime," gr-qc/9707062.

[28] T. Jacobson, "Introduction to quantum fields in curved spacetime and the Hawking effect," gr-qc/0308048.

[29] R. Penrose, "Conformal treatment of infinity," in Relativity, groups and topology, C. M. de Witt and B. de Witt, eds. Gordon \& Breach, New York, 1964.

[30] R. Penrose, "Zero rest mass fields including gravitation: Asymptotic behavior," Proc. Roy. Soc. Lond. A284 (1965) 159.

[31] L. Fidkowski, V. Hubeny, M. Kleban, and S. Shenker, "The black hole singularity in ads/cft," JHEP 02 (2004) 014, hep-th/0306170.

[32] W. Israel, "Event horizons in static vacuum space-times," Phys. Rev. 164 (1967) 1776-1779.

[33] G. W. Gibbons, D. Ida, and T. Shiromizu, "Uniqueness and non-uniqueness of static black holes in higher dimensions," Phys. Rev. Lett. 89 (2002) 041101, hep-th/0206049.

[34] M. Heusler, "Stationary black holes: Uniqueness and beyond," Living Rev. Rel. 1 (1998) 6.

[35] R. Emparan and H. S. Reall, "A rotating black ring in five dimensions," Phys. Rev. Lett. 88 (2002) 101101, hep-th/0110260.

[36] S. W. Hawking and G. F. R. Ellis, The large scale structure of space-time. Cambridge Univ. Press, Cambridge, UK, 1973.

[37] B. Carter, "Black hole equilibrium states," in Black Holes, C. DeWitt and B. S. DeWitt, eds. Gordon \& Breach, New York, 1973.

[38] P. K. Townsend, "Black holes," gr-qc/9707012

[39] S. W. Hawking, "Gravitational radiation from colliding black holes," Phys. Rev. Lett. 26 (1971) 1344-1346. 
[40] J. D. Bekenstein, "Black holes and entropy," Phys. Rev. D 7 (1973) 2333-2346.

[41] J. Bardeen, B. Carter, and S. W. Hawking, "The four laws of black hole mechanics," Commun. Math. Phys. 31 (1973) 161.

[42] T. Wiseman, "Static axisymmetric vacuum solutions and non-uniform black strings," Class. Quant. Grav. 20 (2003) 1137-1176, hep-th/0209051.

[43] R. Emparan, "Rotating circular strings, and infinite non-uniqueness of black rings," JHEP 03 (2004) 064, hep-th/0402149.

[44] A. Ashtekar et. al., "Isolated horizons and their applications," Phys. Rev. Lett. 85 (2000) 3564-3567, gr-qc/0006006.

[45] R. M. Wald, "Black hole entropy in the Noether charge," Phys. Rev. D 48 (1993) 3427-3431, gr-qc/9307038.

[46] V. Iyer and R. M. Wald, "Some properties of Noether charge and a proposal for dynamical black hole entropy," Phys. Rev. D50 (1994) 846-864, gr-qc/9403028.

[47] D. Sudarsky and R. M. Wald, "Extrema of mass, stationarity, and staticity, and solutions to the Einstein Yang-Mills equations," Phys. Rev. D 46 (1992) $1453-1474$.

[48] T. Jacobson and R. C. Myers, "Black hole entropy and higher curvature interactions," Phys. Rev. Lett. 70 (1993) 3684-3687, hep-th/9305016.

[49] G. Lopes Cardoso, B. de Wit, and T. Mohaupt, "Corrections to macroscopic supersymmetric black-hole entropy," Phys. Lett. B451 (1999) 309-316, hep-th/9812082.

[50] G. Lopes Cardoso, B. de Wit, and T. Mohaupt, "Deviations from the area law for supersymmetric black holes," Fortsch. Phys. 48 (2000) 49-64, hep-th/9904005.

[51] G. Lopes Cardoso, B. de Wit, and T. Mohaupt, "Macroscopic entropy formulae and non-holomorphic corrections for supersymmetric black holes," Nucl. Phys. B567 (2000) 87-110, hep-th/9906094.

[52] J. M. Maldacena, A. Strominger, and E. Witten, "Black hole entropy in M-theory," JHEP 12 (1997) 002, hep-th/9711053.

[53] C. Vafa, "Black holes and Calabi-Yau threefolds," Adv. Theor. Math. Phys. 2 (1998) 207-218, hep-th/9711067.

[54] H. Ooguri, A. Strominger, and C. Vafa, "Black hole attractors and the topological string," hep-th/0405146. 
[55] T. Jacobson, G. Kang, and R. C. Myers, "Increase of black hole entropy in higher curvature gravity," Phys. Rev. D 52 (1995) 3518-3528, gr-qc/9503020.

[56] H. Minkowski, "Space and time," 1908. Address at the 80th Assembly of German Natural Scientists and Physicians.

[57] R. E. Peierls, "The commutation laws of relativistic field theory," Proc. Roy. Soc. (London) A214 (1952) 143.

[58] B. S. Kay and R. M. Wald, "Theorems on the uniqueness and thermal properties of stationary, nonsingular, quasifree states on space-times with a bifurcate Killing horizon," Phys. Rept. 207 (1991) 49-136.

[59] R. Kubo, "Statistical mechanical theory of irreversible processes. 1. General theory and simple applications in magnetic and conduction problems," J. Phys. Soc. Jap. 12 (1957) 570-586.

[60] P. C. Martin and J. S. Schwinger, "Theory of many particle systems. I," Phys. Rev. 115 (1959) 1342-1373.

[61] G. L. Sewell, Quantum theory of collective phenomena. Oxford Univ. Press, Oxford, UK, 1986.

[62] W. G. Unruh, "Notes on black hole evaporation," Phys. Rev. D 14 (1976) 870.

[63] J. J. Bisognano and E. H. Wichmann, "On the duality condition for a Hermitian scalar field," J. Math. Phys. 16 (1975) 985-1007.

[64] J. J. Bisognano and E. H. Wichmann, "On the duality condition for quantum fields," J. Math. Phys. 17 (1976) 303-321.

[65] G. L. Sewell, "Quantum fields on manifolds: PCT and gravitationally indiced thermal states," Ann. Phys. 141 (1982) 201.

[66] G. W. Gibbons and M. J. Perry, "Black holes and thermal Green's functions," Proc. Roy. Soc. Lond. A358 (1978) 467-494.

[67] S. W. Hawking, "The path-integral approach to quantum gravity," in General Relativity: An Einstein Centenary Survey, S. W. Hawking and W. Israel, eds., pp. 746-789. Cambridge Univ. Press, Cambridge, UK, 1979.

[68] G. W. Gibbons, "Euclidean quantum gravity: the view from 2002," in The Future of Theoretical Physics and Cosmology, G. W. Gibbons, E. P. S. Shellard, and S. J. Rankin, eds. Cambridge Univ. Press, Cambridge, UK, 2003.

[69] M. Banados, C. Teitelboim, and J. Zanelli, "Black hole entropy and the dimensional continuation of the gauss-bonnet theorem," Phys. Rev. Lett. 72 (1994) 957-960, gr-qc/9309026. 
[70] S. W. Hawking, G. T. Horowitz, and S. F. Ross, "Entropy, area, and black hole pairs," Phys. Rev. D 51 (1995) 4302-4314, gr-qc/9409013

[71] G. W. Gibbons and S. W. Hawking, "Action integrals and partition functions in quantum gravity," Phys. Rev. D 15 (1977) 2752-2756.

[72] V. Iyer and R. M. Wald, "A comparison of Noether charge and Euclidean methods for computing the entropy of stationary black holes," Phys. Rev. D52 (1995) 4430-4439, gr-qc/9503052.

[73] S. F. Ross, "Black hole pair creation," in The Future of Theoretical Physics and Cosmology, G. W. Gibbons, E. P. S. Shellard, and S. J. Rankin, eds.

Cambridge Univ. Press, Cambridge, UK, 2003.

[74] J. M. Maldacena, "The large N limit of superconformal field theories and supergravity," Adv. Theor. Math. Phys. 2 (1998) 231-252, hep-th/9711200.

[75] S. S. Gubser, I. R. Klebanov, and A. M. Polyakov, "Gauge theory correlators from non-critical string theory," Phys. Lett. B428 (1998) 105-114, hep-th/9802109.

[76] E. Witten, "Anti-de Sitter space and holography," Adv. Theor. Math. Phys. 2 (1998) 253-291, hep-th/9802150.

[77] S. S. Gubser, I. R. Klebanov, and A. W. Peet, "Entropy and temperature of black 3-branes," Phys. Rev. D 54 (1996) 3915-3919, hep-th/9602135.

[78] A. Strominger, "Black hole entropy from near-horizon microstates," JHEP 02 (1998) 009, hep-th/9712251.

[79] J. D. Brown and M. Henneaux, "Central charges in the canonical realization of asymptotic symmetries: An example from three-dimensional gravity," Commun. Math. Phys. 104 (1986) 207-226.

[80] S. W. Hawking and D. N. Page, "Thermodynamics of black holes in anti-de Sitter space," Commun. Math. Phys. 87 (1983) 577.

[81] E. Witten, "Anti-de Sitter space, thermal phase transition, and confinement in gauge theories," Adv. Theor. Math. Phys. 2 (1998) 505-532, hep-th/9803131.

[82] O. Aharony, J. Marsano, S. Minwalla, K. Papadodimas, and M. Van Raamsdonk, "The Hagedorn/deconfinement phase transition in weakly coupled large $N$ gauge theories," hep-th/0310285.

[83] R. Dijkgraaf, J. M. Maldacena, G. W. Moore, and E. Verlinde, "A black hole farey tail," hep-th/0005003.

[84] G. T. Horowitz and D. Marolf, "A new approach to string cosmology," JHEP 07 (1998) 014, hep-th/9805207. 
[85] V. Balasubramanian, P. Kraus, A. E. Lawrence, and S. P. Trivedi, "Holographic probes of anti-de Sitter space-times," Phys. Rev. D 59 (1999) 104021, hep-th/9808017.

[86] J. M. Maldacena, "Eternal black holes in anti-de-Sitter," JHEP 04 (2003) 021, hep-th/0106112.

[87] P. Kraus, H. Ooguri, and S. Shenker, "Inside the horizon with AdS/CFT," Phys. Rev. D 67 (2003) 124022, hep-th/0212277

[88] T. S. Levi and S. F. Ross, "Holography beyond the horizon and cosmic censorship," Phys. Rev. D 68 (2003) 044005, hep-th/0304150.

[89] L. Fidkowski, V. Hubeny, M. Kleban, and S. Shenker, "The black hole singularity in AdS/CFT," JHEP 02 (2004) 014, hep-th/0306170.

[90] J. Kaplan, "Extracting data from behind horizons with the AdS/CFT correspondence," hep-th/0402066.

[91] V. Balasubramanian and T. S. Levi, "Beyond the veil: Inner horizon instability and holography," Phys. Rev. D 70 (2004) 106005, hep-th/0405048.

[92] D. Brecher, J. He, and M. Rozali, "On charged black holes in anti-de Sitter space," hep-th/0410214.

[93] D. Marolf, "States and boundary terms: Subtleties of Lorentzian AdS/CFT," hep-th/0412032.

[94] S. W. Hawking, "Breakdown of predictability in gravitational collapse," Phys. Rev. D 14 (1976) 2460-2473.

[95] D. N. Page, "Black hole information," hep-th/9305040. 